\title{
Infra-slow modulation of fast beta/gamma oscillations in the mouse visual system
}

\author{
Patrycja Orlowska-Feuer ${ }^{1}$ (D), Annette Elisabeth Allen ${ }^{2}$ (D), Timothy Matthew Brown ${ }^{3}$ (D), \\ Hanna Jowita Szkudlarek ${ }^{1}$ (i), Robert James Lucas ${ }^{3}$ (1) and Riccardo Storchi ${ }^{2}$ (I) \\ ${ }^{1}$ Department of Neurophysiology and Chronobiology, Institute of Zoology and Biomedical Research, Jagiellonian University in Krakow, Krakow, 30-387, \\ Poland \\ ${ }^{2}$ Division of Neuroscience and Mental Health, Faculty of Biology, Medicine and Health, University of Manchester, Manchester, M13 9PT, UK \\ ${ }^{3}$ Centre for Biological Timing, Faculty of Biology, Medicine and Health, University of Manchester, Manchester, M13 9PT, UK
}

Edited by: Ian Forsythe \& Diego Contreras

\begin{abstract}
Key points
- Neurophysiological activity in the subcortical visual system fluctuates in both infra-slow and fast oscillatory ranges, but the level of co-occurrence and potential functional interaction of these rhythms is unknown.

- Analysing dark-adapted spontaneous activity in the mouse subcortical visual system, we find that these two types of oscillation interact uniquely through a population of neurons expressing both rhythms.

- Genetic ablation of rod/cone signalling potentiates infra-slow and abolishes fast beta/gamma oscillations while genetic ablation of melanopsin substantially diminishes the interaction between these two rhythms.

- Our results indicate that in an intact visual system the phase of infra-slow modulates fast beta/gamma oscillations.

- Thus one possible impact of infra-slow oscillations in vision is to guide visual processing by interacting with fast narrowband oscillations.
\end{abstract}

\begin{abstract}
Infra-slow $(<0.02 \mathrm{~Hz})$ and fast beta/gamma $(20-100 \mathrm{~Hz})$ oscillations in neurophysiological activity have been widely found in the subcortical visual system. While it is well established that fast beta/gamma oscillations are involved in visual processing, the role (if any) of infra-slow oscillations is currently unknown. One possibility is that infra-slow oscillations exert influence by modulating the amplitude of fast oscillations, yet the extent to which these different oscillations arise independently and interact remains unknown. We addressed these questions by recording in vivo spontaneous activity from the subcortical visual system of visually intact mice,
\end{abstract}

Patrycja Orlowska-Feuer received her PhD in Biology from the Jagiellonian University in Krakow, Poland in 2014. Following a postdoctoral position at the Jagiellonian University, she obtained Bekker Fellowship (NAWA, Poland) and subsequently Marie Sklodowska-Curie Individual Fellowship and became a research fellow at the University of Manchester. Since her postgraduate studies, she has been particularly interested in the mechanism(s) and source(s) of infra-slow oscillatory activity in the subcortical visual system with emphasis put on the retina. Her current research focuses on melanopsin contribution to vision formation in diurnal and nocturnal rodents.

Authors' present addresses Patrycja Orlowska-Feuer: Division of Neuroscience and Mental Health, Faculty of Biology, Medicine and Health, University of Manchester M13 9PT, Manchester, UK. Hanna Jowita Szkudlarek: Department of Anatomy and Cell Biology, Medical Science Bldg, University of Western Ontario, London, ON, Canada.

This article was first published as a preprint. Orlowska-Feuer P, Allen AE, Brown TM, Szkudlarek HJ, Lucas RJ, Storchi R. 2020. Infra-slow modulation of fast beta/gamma oscillations in the mouse visual system. bioRxiv. https://doi.org/10.1101/2020.02.24.963124. 
and animals whose retinal network was disrupted by advanced rod/cone degeneration $(r d / r d c l)$ or melanopsin loss $\left(\mathrm{Opn} 4^{-/-}\right)$. We found many neurons expressing only one type of oscillation, and indeed fast oscillations were absent in $r d / r d c l$. Conversely, neurons co-expressing the two oscillations were also common, and were encountered more often than expected by chance in visually intact but not $\mathrm{Opn} 4^{-/-}$mice. Finally, where they co-occurred we found that beta/gamma amplitude was modulated by the infra-slow rhythm. Our data thus reveal that: (1) infra-slow and beta-gamma oscillations are separable phenomena; and (2) that they actively co-occur in a subset of neurones in which the phase of infra-slow oscillations defines beta-gamma oscillations amplitude. These findings suggest that infra-slow oscillations could influence vision by modulating beta-gamma oscillations, and raise the possibility that disruptions in these oscillatory behaviours contribute to vision dysfunction in retinal dystrophy.

(Received 22 April 2020; accepted after revision 5 January 2021; first published online 11 January 2021)

Corresponding authors P. Orlowska-Feuer: Department of Neurophysiology and Chronobiology, Institute of Zoology and Biomedical Research, Jagiellonian University in Krakow, Krakow, 30-387 Poland. Email: patrycja.orlowska@uj.edu.pl,patrycjaanna.orlowska-feuer@manchester.ac.uk

R. Storchi: Division of Neuroscience and Mental Health, Faculty of Biology, Medicine and Health, University of Manchester, Manchester, M13 9PT UK. Email: riccardo.storchi@manchester.ac.uk

\section{Introduction}

Regular infra-slow $(<0.02 \mathrm{~Hz})$ oscillations in neurophysiological activity have been found throughout the mammalian visual system, including the retina (Rodieck \& Smith, 1966), optic chiasm (Cavaggioni, 1968), the dorsal lateral geniculate nucleus (dLGN) (Albrecht \& Gabriel, 1994; Albrecht et al. 1998), ventral lateral geniculate nucleus (vLGN) (Chrobok et al. 2018), olivary pretectal nucleus (OPN) (Szkudlarek et al. 2008, 2012), suprachiasmatic nucleus (SCN) (Aggelopoulos \& Meissl, 2000), intergeniculate leaflet (IGL) (Lewandowski et al. 2000, 2002) and primary visual cortex (Filippov \& Frolov, 2005). Infra-slow oscillatory rhythm features such as frequency and amplitude are modulated by light and depend on retinal activity (Miller \& Fuller, 1992; Albrecht et al. 1998; Lewandowski et al. 2000; Szkudlarek et al. 2012; Orlowska-Feuer et al. 2016a). Nevertheless, despite its ubiquity, the contribution of such rhythmicity, if any, to visual processing is still a matter of debate.

Oscillations at a quite different frequency range (20-100 Hz, termed beta/gamma) are also a feature of the early visual system. Such oscillations have been implicated in different aspects of visual processing ranging from inter-area communication (Fries, 2005) to feature binding (Gray et al. 1989). Recently, we and others also reported that the power of fast beta/gamma oscillations encodes for ambient light intensity and spatially structured changes in luminance (Saleem et al. 2017; Storchi et al. 2017).

Decades of research have highlighted a general organizing principle by which the brain orchestrates oscillations expressed at different timescales. Namely, faster oscillations are 'nested' within slower oscillations so that the power of faster oscillations is controlled by the phase of the slower ones. A remarkable example of this phenomenon is the theta-gamma coupling in the hippocampus that has been shown to underlie spatial navigation and object memory recollection (Lisman \& Jensen, 2013; Gupta et al. 2016). Electroencephalographic recordings in humans indicate that infra-slow oscillations can also modulate faster rhythms (Vanhatalo et al. 2004; Monto et al. 2008). Thus one possible impact of infra-slow oscillations in vision is to guide visual processing by interacting with fast narrowband oscillations.

To test this possibility we recorded from mouse subcortical visual system (dLGN, vLGN and OPN). We found that infra-slow and fast beta/gamma oscillations interact through a population of neurons that co-express both rhythms. Namely in these neurons the phase of infra-slow oscillations modulates the amplitude of gamma oscillations. We then asked whether this interaction required intact vision by repeating the same experiments in animals with genetic ablation of rods+cones or melanopsin photoreception. We found that rod+cone loss abolished fast oscillations, but left infra-slow rhythm intact, whereas both oscillations were retained in melanopsin knockouts but their co-expression was substantially reduced. Together these results confirm that infra-slow and fast oscillations are separable phenomena reflecting different network states, and also that in physiological conditions their co-expression in the same neurons is an active consequence of a particular network state that is disrupted by melanopsin loss.

\section{Methods}

\section{Ethical approval}

All procedures involving animals were carried out in accordance with regulations and standards of the European Community Council Directive of 24 November 
1986 (86/609/EEC), approvals of the institutional ethics committee (70/8918) and the UK Home Office and standard and regulations described by Grundy (Grundy, 2015).

The following report is in part a re-analysis of previously collected data that has been partially published elsewhere (Brown et al. 2010; Allen et al. 2011).

\section{Animals}

Experiments were performed on genetically modified mice which were bred in the animal facility of the Faculty of Life Sciences at Manchester University, UK. Animals were housed under standard conditions (60\% of humidity and food and water at libitum) with $12: 12 \mathrm{~h}$ light/dark cycle. Animals were 3- to 5-month-old males with three different genetic backgrounds: Opn $1 m w^{R}$ mice (C57BL/6:129sv strain) had all types of photoreceptors functional; rodless and coneless $(r d / r d c l)$ mice (C3H strain) lacked rods and cones and $\mathrm{Opn}^{4^{-/-}}$mice (C57BL/6:129sv strain) lacked melanopsin. Opn1mw ${ }^{R}$ mice had the medium wavelength-sensitivity (MWS) opsin replaced by human red opsin. This manipulation shifts cone spectral sensitivity but has no other known effects on retinal functions and connectivity (Smallwood et al. 2003; Jacobs \& Williams, 2007). Therefore, since the manipulation is inconsequential for this study, Opn $1 m w^{R}$ mice were used as 'wild type' animals. All animals were removed from their home cages at the beginning of the projected day and the electrophysiological recordings spanned the mid-portion of the day. In total 61 animals were used: 22 of $O p n 1 m w^{R}, 22$ of $O p n 4^{-/-}$and 17 of $r d / r d$ cl mice.

\section{Surgery}

All surgical procedures were conducted under deep urethane anaesthesia (1.55 $\mathrm{g} \mathrm{kg}^{-1}, 30 \% \mathrm{w} / \mathrm{v}$; Sigma-Aldrich, Munich, Germany). Animals were injected intraperitoneally and the depth of anaesthesia was ascertained by lack of withdrawal and ocular reflexes. If necessary, animals were supplemented with $10 \%$ of the initial dose of urethane; however, that was never done during the recordings. Body temperature was automatically maintained at $37 \pm 0.5^{\circ} \mathrm{C}$ by the thermistor with a feedback-controlled heating pad. Anesthetized mice were carefully placed in a stereotaxic frame via ear bars (SR-15M, Narishige International Limited, London, UK). Skin and all soft tissues covering the skull were removed exposing coronal, sagittal and lambdoid sutures. Next, bregma point was determined and craniotomy was performed above the OPN or LGN. The coordinates were assessed based on a stereotaxic brain atlas for mice (Paxinos \& Franklin, 2004) and were AP, -2.8; LM, 0.9;
$\mathrm{DV},-2$ to $-2.8 \mathrm{~mm}$ and AP, -2.5 ; LM, 2.2; DV, 2.5 to $3.5 \mathrm{~mm}$ for the OPN and LGN, respectively.

\section{Electrophysiology}

A 32-channel multiunit electrode array (A4X8-5 mm-50-200-413; NeuroNexus Technologies, Dallas, TX, USA) was used to record neuronal activities from the pretectum and LGN area. It consisted of 4 silicon shanks (spaced $200 \mu \mathrm{m}$ ), each with 8 recordings sites spaced vertically at $50 \mu \mathrm{m}$. Neuronal signals were acquired using Recorder64 system (Plexon, Dallas, TX, USA). Signals were amplified $(3000 \times)$, filtered $(300 \mathrm{~Hz})$ and digitized at $40 \mathrm{kHz}$. All data were saved on a computer hard disc for further analysis.

A $4 \times 8$ electrode array was introduced to a $350 \times 600 \mu \mathrm{m}$ area of the pretectum or LGN and recordings were taken regardless of types of recorded activities (either oscillatory or non-oscillatory). All experiments were conducted in dark-adapted conditions provided by coverage of the Faraday cage with light-impermeable material (effective photon flux $\sim$ $9 \log$ photons $\mathrm{cm}^{-2} \mathrm{~s}^{-1}$ ). Moreover, recordings in some animals were not limited to one penetration.

\section{Histological verification}

Prior to the first insertion, the electrode was dipped in fluorescent dye (Cell Tracker CM-Dil, Invitrogen Ltd, UK) in order to verify position of all recording places. At the end of the experiment animals were intracardially perfused with a buffered physiological saline followed by $4 \%$ paraformaldehyde in $0.1 \mathrm{~m}$ phosphate buffer $(\mathrm{pH}=7.2)$. Brains were removed from the skull and postfixed overnight in the same fixative at $4^{\circ} \mathrm{C}$. Cryoprotected brains (immersion in $30 \%$ sucrose solution for 2-3 days) were cut on a freezing microtome at a coronal plane. Sequential sections (100 $\mu \mathrm{m}$ thick) with CM-Dil depositions were mounted on gelatin-coated glass slides. Sections were carefully inspected, photographed and ascribed to a related coronal plain of the brain according to the stereotaxic atlas of a mouse brain (Paxinos \& Franklin, 2004). Expression of the parvalbumin (calcium binding protein) was used as the marker of OPN neurons (for the precise description of the method see Allen et al. 2011). The optic tract, hippocampus and third ventricle were used as landmarks to determine the LGN borders.

\section{Statistics}

Spike sorting. Multi-unit recordings were processed using Offline Sorter (version 2.8.8; Plexon) or Spike2 (version 6.08). After removal of cross-channel artifacts, each channel was analysed separately. Single units were 
detected and categorized based on spike waveform via principal component analysis (PCA) and related statistic (Fig. $2 A$ and $B$ ). Moreover, the inter-spike interval (ISI) histograms were computed to monitor unit refractory period. Cross-correlograms for all isolated units were examined to ensure that each unit was only included once in the further analysis (probability of synchronous firing $<0.01$ ). In total we detected 2606 neurons and 2372 (those with firing rates $>0.02 \mathrm{~Hz}$ ) were further analysed.

Detection of infra-slow activity. Regular oscillations in the infra-slow range have been previously shown in several publications (Miller \& Fuller, 1992; Albrecht \& Gabriel, 1994; Lewandowski et al. 2000; Szkudlarek et al. 2008; Orlowska-Feuer et al. 2016a; Tsuji et al. 2016; Chrobok et al. 2018). The frequency of those oscillations has been shown to be $<0.02 \mathrm{~Hz}$ even when long recording epochs ( $>1$ h, Orlowska-Feuer et al. 2016a; Chrobok et al. 2018) were considered. However, in such works the assessment was largely done qualitatively based on visual inspection of firing rate time series, autocorrelations and power spectra. Here, we devised a procedure to classify those units automatically. We first observed that, for units expressing the most regular infra-slow oscillations, their autocorrelation could be well fitted by using the following equation:

$A C_{\text {flt }}(t)=A_{1} \cos \left(2 \pi t / T_{1}\right) \mathrm{e}^{-t / \tau_{1}}+A_{2} \cos \left(2 \pi t / T_{2}\right) \mathrm{e}^{-t / \tau_{2}}$,

where $\mathrm{e}^{-t / \tau_{i}}$ captures the reduction in autocorrelation as function of time lag $t$ and $\cos \left(2 \pi t / T_{i}\right)$ captures regular oscillations (Fig. $1 A$ and $B$ ). The dominant oscillation was defined so that for all units $A_{1} \tau_{1}>A_{2} \tau_{2}$. The ratio $T_{1} / T_{2}$ clustered around 0.5 and 2 indicating that the second oscillation typically approximates a harmonic of the dominant oscillation (Fig. 1C). For classification we focused on the dominant oscillation. In order to classify a unit as an infra-slow we used the following criteria:

(1) Consistent with previous literature, the period T1 has to be within the 50 s and 450 s (Miller \& Fuller, 1992; Lewandowski et al. 2000; Filippov et al. 2004; Szkudlarek et al. 2008).

(2) The relation $\tau_{1} / T_{1}$ between time constant and oscillation period has to be above a fixed threshold $(=0.6)$ in order to reveal regular oscillations (see Fig. $1 D-F)$.

(3) The autocorrelation model of eqn (1) has to reduce (by $>22.5 \%$ ) the relative fitting error over a simpler model described by $A C_{\text {flt simple }}(t)=A \mathrm{e}^{-t / \tau}$.

Since no clear indication of bimodality was present in the data (see Fig. $1 G$ and $H$ ) numerical thresholds for criteria (2) and (3) were derived empirically to match manual qualitative classification on a subset of data.
However, the full dataset clearly shows a neat separation between units that were classified as infra-slow and those that were not (Fig. 1I). Consistently with results obtained in the cat LGN by (Teich et al. 1997) both, units classified as infra-slow and those that were not, exhibited fluctuations in the infra-slow range (Fig. 1J).

Detection of fast beta/gamma activity. We first calculated the power spectral density (PSD) for each unit (Fig. $1 \mathrm{~K}$ ) by using the Welch method (time bin $=1 \mathrm{~s}$ across the whole $900 \mathrm{~s}$ recording epochs). For this analysis we applied Hamming windows to $512 \mathrm{~s}$ epochs and $50 \%$ overlap between epochs (see MATLAB function pwelch.m). In order to identify narrowband peaks in the beta/gamma range we de-trended the PSD by using a one dimensional median filter (Fig. $1 K$; filter order $=20$; see MATLAB function medfilt $1 . m$ ). The threshold for narrowband peaks was then calculated as equalling 5 times the standard deviation of the de-trended PSD (Fig. $1 K$ ). The units classified as expressing beta/gamma oscillations showed a clear narrow peak in the PSD (Fig. 1L).

Estimation of cross-correlation index. A cross-correlation function was estimated between all pairs of units simultaneously recorded. For each pair a cross-correlation index was then obtained by calculating the median of the cross-correlation function in the interval $(-10,10)$ seconds. To test whether this index was significantly different from zero we applied a sign-test at the distribution of cross-correlation indexes across the selected units.

Estimation of coupling between infra-slow and fast beta/gamma oscillations. Spike timestamps were binned at $1 \mathrm{~s}$ for infra-slow and $5 \mathrm{~ms}$ for fast beta/gamma to obtain spike counts. Those counts were filtered by using zero-phase Kaiser filters (infra-slow: low-pass filter with cut-off at $2.5 / T_{1} \mathrm{~Hz}$ and stop-band at $5 / T_{1} \mathrm{~Hz}$; fast beta/gamma: band-pass filter with cut-off at 0.8 and 1.2 times the peak frequency and stop-band at 0.6 and 1.4 times). A Hilbert transformation was then applied to the spike counts filtered in the infra-slow range and the phase of these signals was clipped in the range $(-\pi, \pi)$. Finally, the phase of infra-slow spike counts was used to divide fast beta/gamma band spike counts into the intervals $((0, \pi / 2) ;(\pi / 2, \pi) ;(0,-\pi / 2) ;(-\pi / 2,-\pi))$. In order to test against the possibility that the amplitude of fast beta/gamma oscillations is modulated by the phase of infra-slow oscillations, we compared the amplitude of these oscillations in the range $(-\pi / 2, \pi / 2)$ with the amplitude observed in the complementary range by using a non-parametric sign-test. 

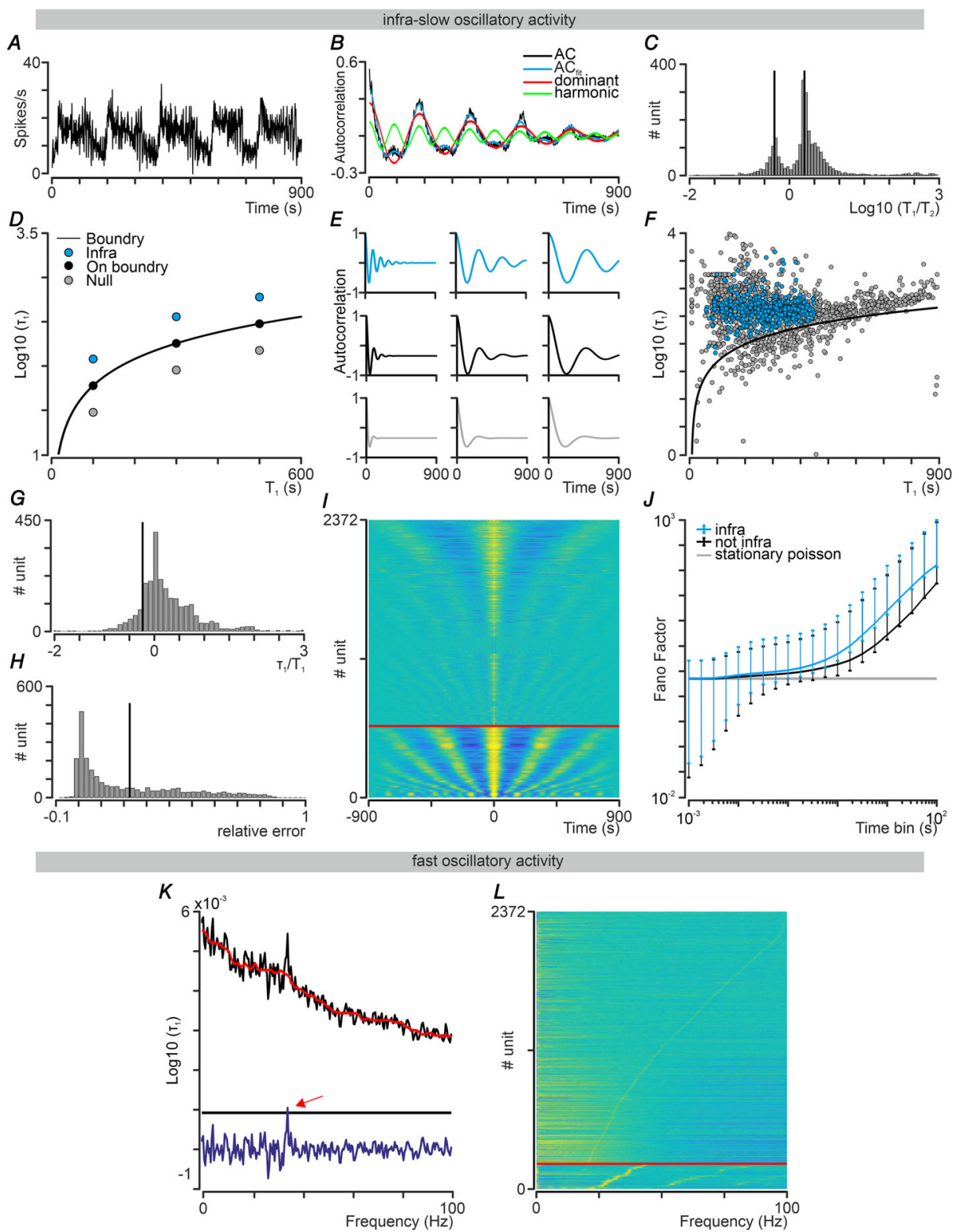

Figure 1. Infra-slow and fast beta/gamma criteria for classification

$A$, firing pattern of a representative unit expressing regular infra-slow oscillations. $B$, the autocorrelogram (black) for unit in panel $A$ can be fitted with a simple model expressed in eqn (1) (blue) and composed of a dominant (red) and a sub-dominant (harmonic, green) component. $C$, histogram of the ratio between period of the dominant and sub-dominant components in eqn (1) for the whole dataset $(n=2372)$. $D$, visual representation of the second classification criterion for classifying unit as infra-slow: the ratio $\tau_{1} / T_{1}$ between time constant and oscillation period has to be above a fixed threshold (=0.6). Blue dots represent surrogate units meeting the criterion, grey dots represent units that don't meet the criterion and black dots units located on the boundary. $E$, autocorrelograms for the units in panel $D$. $F$, same as panel $D$ for all the units in the dataset $(n=2372)$. Blue dots indicate units that meet all the three criteria for infra-slow classification. $G$ and $H$, histograms for $\tau_{1} / T_{1}$ ratio $(G)$ and relative error $(H)$ for all units in the dataset $(n=2372)$. I, cross-correlogram for all units in the dataset $(n=2372)$. Units classified as infra-slow are displayed at the bottom below the red line. All units are re-ordered according to $T_{1}$ parameter. J, Fano factor as function of time window for spike counts for infra-slow units (blue; mean and 0.01-0.99 confidence interval) and for units that do not express regular infra-slow oscillations (black; mean and 0.01-0.99 confidence interval). Both groups deviate from a stationary Poisson process (grey line). $K$, detection of narrowband oscillations in the beta/gamma range. The PSD for a representative unit (black) is rectified by using a median filter (red). A threshold of 5SD is applied to the rectified PSD (blue) to detect the narrowband peak. L, PSD for all units in the dataset $(n=2372)$. Units classified as expressing fast beta/gamma oscillations are displayed at the bottom below the red line. All units are re-ordered according to oscillations frequency (note the narrowband peak is indicated by the red arrow). [Colour figure can be viewed at wileyonlinelibrary.com] 
Table 1. Permutation test

\begin{tabular}{|c|c|c|c|c|c|c|c|}
\hline \multicolumn{4}{|c|}{ Original table } & \multicolumn{4}{|c|}{ Permutation table } \\
\hline Unit no. & Infra-slow & Fast & Co-expression & Unit no. & Infra-slow & Fast & Co-expression \\
\hline 1 & 1 & 0 & 0 & 1 & 1 & 1 & 1 \\
\hline 2 & 1 & 1 & 1 & 2 & 1 & 0 & 0 \\
\hline 3 & 0 & 0 & 0 & 3 & 0 & 0 & 0 \\
\hline 4 & 0 & 0 & 0 & 4 & 0 & 1 & 0 \\
\hline 5 & 1 & 1 & 1 & 5 & 1 & 0 & 0 \\
\hline 6 & 1 & 1 & 1 & 6 & 1 & 0 & 0 \\
\hline 7 & 0 & 0 & 0 & 7 & 0 & 1 & 0 \\
\hline 8 & 1 & 0 & 0 & 8 & 1 & 0 & 0 \\
\hline 9 & 0 & 0 & 0 & 9 & 0 & 0 & 0 \\
\hline Total coexp. & & & 3 & Total coexp. & & & 1 \\
\hline
\end{tabular}

The original table contains the data where infra-slow and fast oscillatory expression was detected, the co-expression column tags the units where infra-slow and fast oscillations were co-expressed. The total number of those units is reported as Total coexp. In the permutation table a random permutation has been applied to the fast column resulting in a different number of units co-expressing both rhythms.

Permutation test. A permutation test was used to test the null hypothesis that occurrence of fast and infra-slow oscillations at the level of individual units were statistically independent events. First, we counted the actual number of units expressing both fast and infra-slow rhythms. Then, we compared those numbers with a null distribution obtained using the following simple permutation technique. First, for each recording, we generated a binary $N$-by-2 matrix where each row represented an individual unit, the first column reported presence (or absence) of infra-slow oscillations (presence $=1$; absence $=0$ ) and the second column presence (or absence) of fast oscillations. Then, in order to break statistical dependence between these oscillations, we performed a random permutation of one of those columns. Finally, we calculated the number of units co-expressing both rhythms (equivalent to a logical AND). Since different realization of a random permutation can produce different results we estimated the null distribution by repeating this procedure 100,000 times. An example is shown in Table 1.

In the real data the firing rate distribution is different between units expressing infra-slow, fast beta/gamma or no oscillations (see Figs $4 G$ and $6 G$ ). Therefore, the number of units co-expressing infra-slow and fast beta/gamma ( $\left.N_{\text {coexpress }}\right)$ expected by chance would equal:

$$
\begin{gathered}
N_{\text {coexpress }}=N \sum_{r=0}^{R \max } P(\text { beta } / \text { gamma } \mid R=r) \\
P(\text { infra } \mid R=r) P(R=r),
\end{gathered}
$$

where $N$ represents the total number of units and $R_{\max }$ the maximum firing rate. Thus, the random permutation procedure needs to preserve the distributions
$P($ beta $/$ gamma $R=r)$ and $P($ infra $R=r)$. We implemented this constraint by grouping units into different classes of firing rates and permuting only across units within the same class. Moreover, in order to also exclude confounding effect of different anatomical structures, random permutations were only applied among units recorded from the same structure.

Statistical comparisons. Statistical comparisons of the means $\pm \mathrm{SD}$ were performed using the non-parametrical Mann-Whitney test, the sign test and the Kruskal-Wallis test (followed by Dunn's multiple comparison test). The sampling distribution was verified with a $\chi^{2}$ test. A probability error of $P<0.05$ was regarded as significant. All data were visualized and statistically examined using GraphPad (version 4.02; GraphPad Software), NeuroExplorer (version 5; Nex Technologies), Statistica software (StatSoft, Inc., Tulsa, Oklahoma, USA) and scripts written in MATLAB (R2019a).

Code accessibility. The original dataset in .csv format and MATLAB codes for replicating the tests are available online at https://github.com/RStorchi/InfraSlow.

\section{Results}

The main aim of this work was to determine the relationship between infra-slow and fast narrowband oscillations in the mouse visual system. We addressed these questions both in image forming and in non-image forming centres in the brain by focusing on the dLGN (image forming), vLGN and OPN (both non-image forming structures). 
Infra-slow oscillations are widespread in the mouse visual system and co-exist with fast narrowband rhythms

Infra-slow oscillations in the subcortical visual system have been observed in many species including rats, cats and monkeys but not previously in mice. Therefore, we first aimed to verify the occurrence of infra-slow oscillatory activity in the spontaneous neuronal firing in the mouse subcortical visual system. We unbiasedly recorded in the absence of visual input (in darkness) from the dLGN, vLGN and OPN in wild-type mice.

Autocorrelation analysis revealed that $\sim 30 \%$ of all recorded units expressed an infra-slow oscillatory rhythm $(0.02-0.0022 \mathrm{~Hz})$ in spiking activity, resembling previously reported oscillations in the rat subcortical visual system (Albrecht \& Gabriel, 1994; Lewandowski et al. 2000; Szkudlarek et al. 2008; Chrobok et al. 2018). In Fig. 2C-E examples of rhythmically firing units in the dLGN, vLGN and OPN are presented. The mean frequency of observed oscillations ranged between 0.0022 and $0.018 \mathrm{~Hz}$ and did not differ between structures (Kruskal-Wallis test, $\mathrm{K}-\mathrm{W}$ statistics $=0.8394$, $P=0.6572$; Fig. $2 G$ ). The proportion of infra-slow oscillatory neurons was also statistically indistinguishable among these nuclei $(28 \%, 22 \%$ and $30 \%$ in the dLGN, vLGN and OPN, respectively; $\chi^{2}$ test, $P=0.3190$, $\chi^{2}=2.285$, df $=2$, Fig. $3 E$ ). In the same dataset described in Fig. $2 C-G$ we found that a fraction of neurons expressed fast narrowband oscillations in the beta/gamma range $(20-100 \mathrm{~Hz}$; Fig. $2 \mathrm{H}-J)$. The power spectra density (PSD) analysis and multimodal distribution of peaks at the level of ISI histograms of spontaneous spike activity revealed that $11 \%(\mathrm{dLGN}), 15 \%$ (vLGN) and $33 \%$ (OPN) of all recorded units expressed such fast oscillations under dark-adapted condition. The frequency of these oscillations averaged at $33.43 \pm 10.39 \mathrm{~Hz}$ (dLGN), $30.68 \pm 6.16 \mathrm{~Hz}$ (vLGN) and $34.91 \pm 5.09 \mathrm{~Hz}$ (OPN). In contrast to infra-slow oscillatory activity, the frequency and proportion of fast oscillating units differed between structures (Kruskal-Wallis test, $P=0.0271, \mathrm{~K}-\mathrm{W}$ statistic $=7.213$, Dunn's multiple comparison test; $\chi^{2}$ test, $P<0.0001, \chi^{2}=30.39, \mathrm{df}=2$; Figs $2 J$ and $3 E$ ).

Based on that data (in total 640 cells from 30 recording sites from 22 wild-type animals) we were able to describe four types of spontaneous activity (in darkness) in the sub-cortical visual system of visually intact mice: infra-slow or fast oscillatory activity alone; co-expression of both rhythms; and no oscillatory activity in the above ranges (Fig. 3A-E). Co-expression of infra-slow and fast oscillations were found in 5\%,6\% and $15 \%$ of all dLGN, vLGN and OPN neurons, respectively $\left(\chi^{2}\right.$ test, $P=0.0022, \chi^{2}$ value $=12.24, \mathrm{df}=2$, Fig. $3 E$ ). Importantly, all four types of neurons (infra-slow, fast, co-expressing both rhythms and none of them) were found simultaneously in 53\% of the recording sessions, indicating that their emergence cannot be trivially explained by global fluctuations in neuronal activity (Fig. 3F). In all the structures considered the expression of fast beta/gamma was limited to a minority of units. Simultaneous LFP recordings, reflecting population activity from all units, did not exhibit such narrowband oscillations (Fig. $3 G$ and $H$ ).

Firing rate of neurons expressing only infra-slow were significantly correlated between each other in all the structures investigated (except the OPN, Table 2 for $\left.O p n 1 m w^{R}\right)$ as revealed by the cross-correlation analysis (see Methods section). Significant correlations were also observed in neurons co-expressing both infra-slow and fast oscillations (Table 2 for Opn $1 m w^{R}$ ). Moreover, these two populations were significantly correlated with each other (Table 2 for Opn1mw $w^{R}$ ), suggesting that a single infra-slow rhythm coordinates firing rate in both populations.

Fast beta/gamma oscillations are modulated in the infra-slow range by diffuse co-expression of both rhythms at single unit level

We next aimed to investigate the possibility that infra-slow oscillations modulate fast beta/gamma oscillations. Specifically, we asked how and to what extent the phase of infra-slow oscillations could modulate the amplitude of fast beta/gamma oscillations.

We first investigated the possibility that this phenomenon could occur between two distinct neuronal populations, one expressing only infra-slow the other only fast beta/gamma. For each recording and brain structure, we calculated the phase of infra-slow and the amplitude of fast beta/gamma by separately pooling infra-slow and fast beta/gamma units and then by applying a frequency filter and a Hilbert transform to each population (see Methods for details). This analysis revealed that neurons expressing only fast oscillations were largely unaffected by the population-level infra-slow oscillations (Fig. $4 \mathrm{~A}$ and $B$ ). We then asked whether this modulation could instead occur within the same population of neurons. Therefore we repeated the same analysis on populations co-expressing both infra-slow and fast beta gamma oscillations. We found that within these populations fast beta/gamma rhythms were strongly modulated according to the phase of infra-slow oscillations (Fig. $4 D$ and E). These results were consistent across the whole dataset, indicating that co-expression of both rhythms at the level of individual unit is required for infra-slow modulation of fast narrowband oscillations (Fig. $4 C$ and $F$ ).

Finally, we measured the extent to which the expressions of infra-slow and fast oscillations were coordinated at population level. 

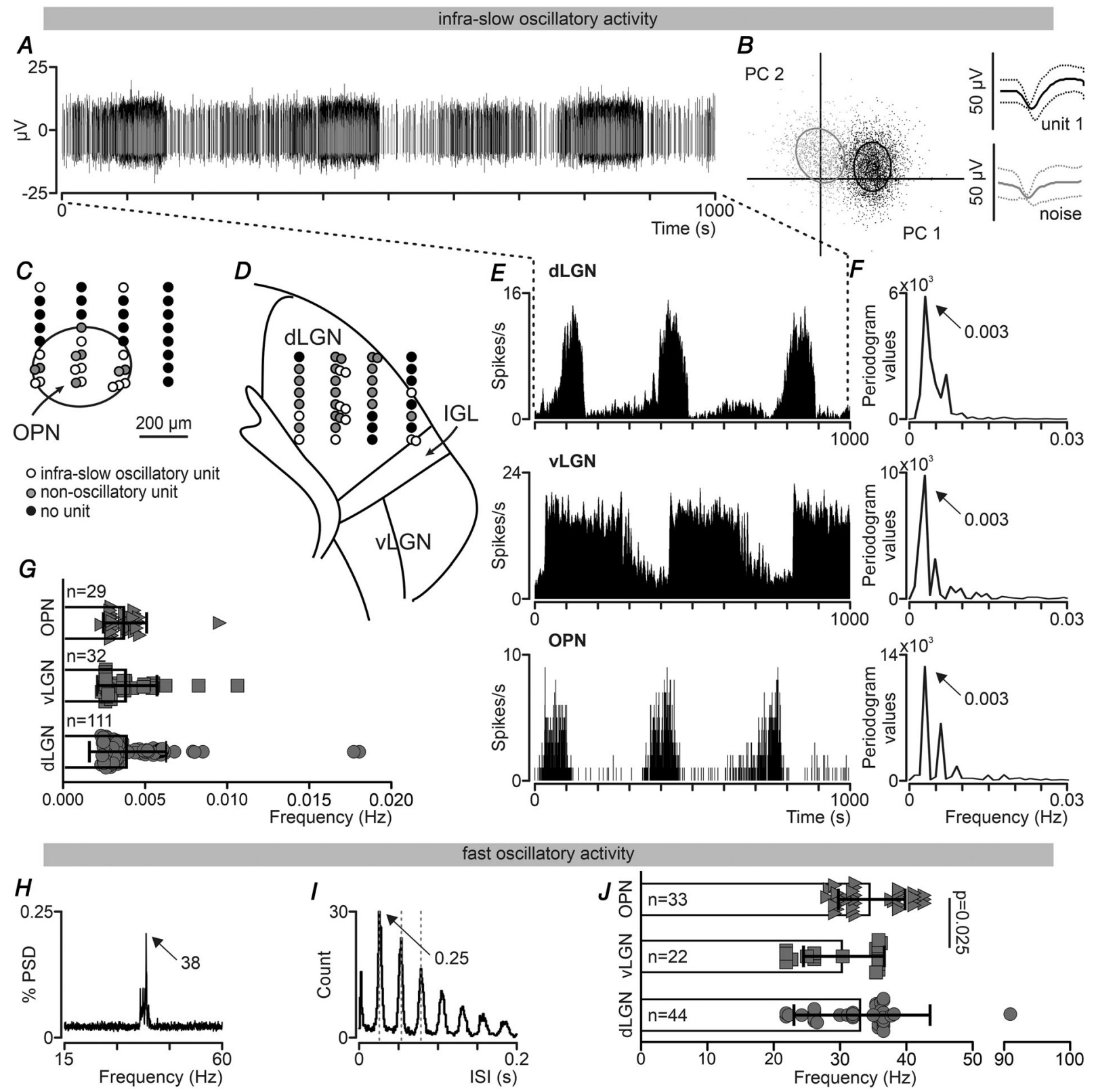

Figure 2. The presence of infra-slow and fast beta/gamma oscillatory activities in the mouse subcortical visual system

$A$, representative trace recorded in the dLGN revealing the infra-slow oscillatory activity in the dark-adapted spontaneous activity. $B$, waveforms were clustered and sorted into units based on principal component analysis (PCA). $C$ and $D$, a schematic representation of the placement of one $4 \times 8$ electrode (= 32 recording channels) showing the distribution of recorded units in the OPN $(C)$ and LGN $(D)$ and whether they were oscillatory (colour coded). $E$, representative firing rate histograms (bin size $=1 \mathrm{~s}$ ) of spontaneous, dark-adapted activity of infra-slow oscillatory cells in the dLGN, vLGN and OPN (labelled above the histograms, not simultaneously recorded). F, FFT analysis performed for the recordings showed in $E$, indicating the infra-slow frequencies. $G$, the mean frequency of infra-slow oscillatory activity in the dLGN, vLGN and OPN does not differ between structures (Kruskal-Wallis test, $\mathrm{K}-\mathrm{W}$ statistics $=0.8394, P=0.6572$ ). $\mathrm{H}$, fast beta/gamma oscillatory activity confirmed by PSD analysis. I, ISI histogram (bin size $=0.001 \mathrm{~s}$ ) computed to confirm existence of harmonic modes in beta/gamma range. J, the mean frequency of fast (beta/gamma) oscillatory activity in the dLGN, VLGN and OPN differs between structures (Kruskal-Wallis test, $\mathrm{K}-\mathrm{W}$ statistics $=7.213, P=0.0271$, Dunn's multiple comparison test). 

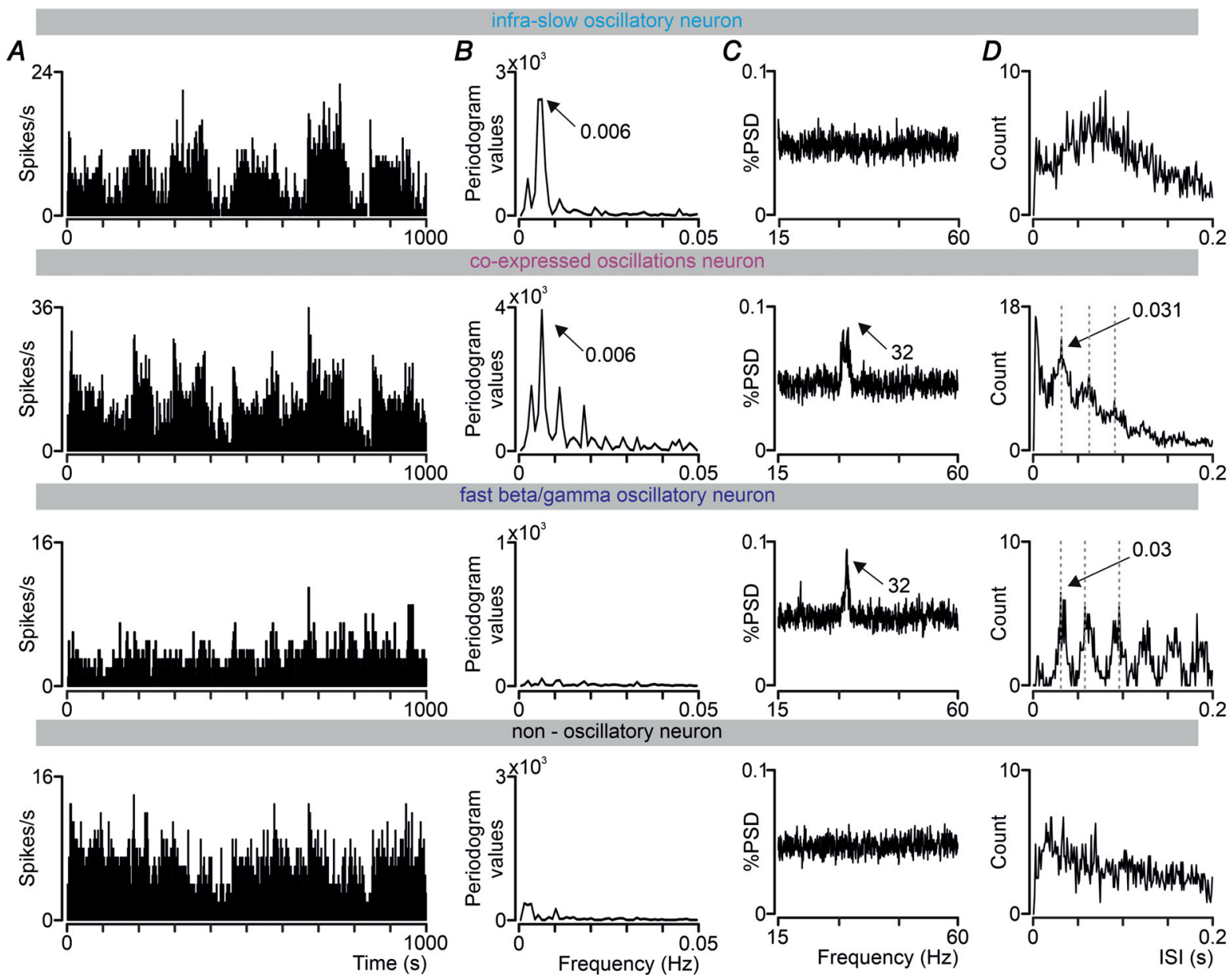
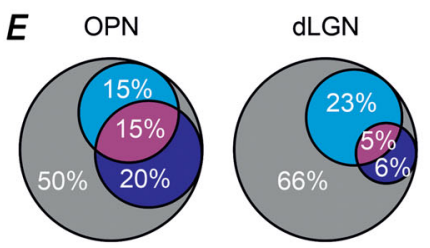

$n=400$

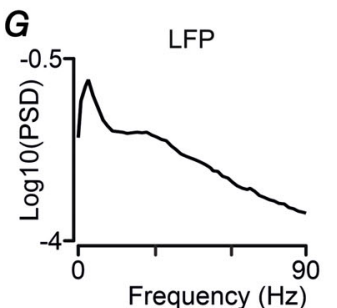

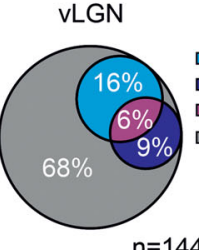

$\square$ infra-slow oscillations fast oscillations

co-expressed oscillations a non-oscillatory units

$F$ Recording sites
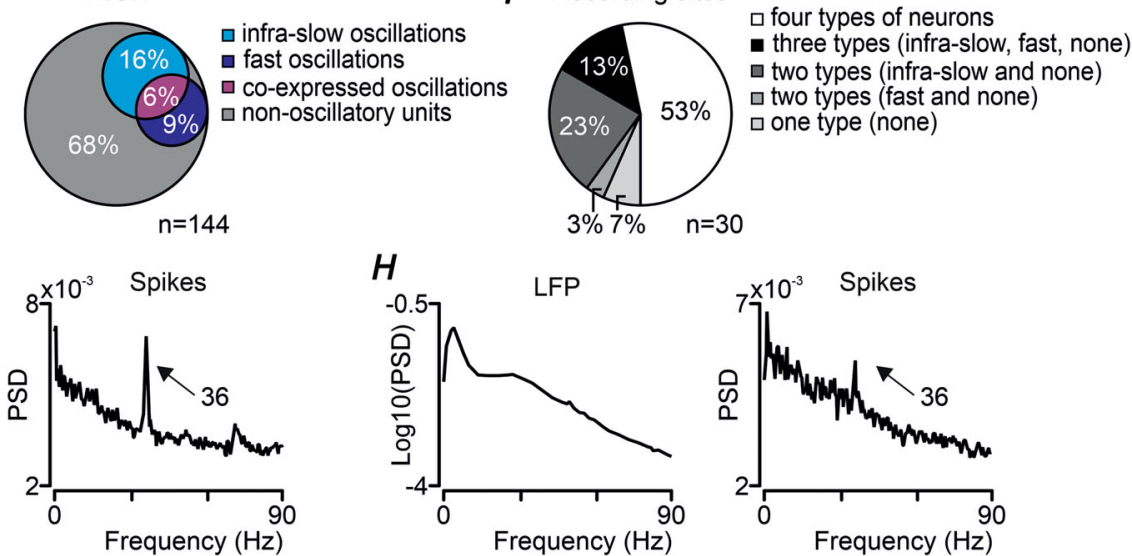

Figure 3. Types of spontaneous activity in the subcortical visual system

$A$, representative firing rate histograms (bin size $=1 \mathrm{~s}$ ) of spontaneous, dark-adapted activity of simultaneously recorded different types of oscillatory cells in the dLGN. $B$ and C, FFT $(B)$ and PSD $(C)$ analysis were performed to classify cells as infra-slow, fast beta/gamma oscillatory, co-expressing both or none of the above frequencies. $D$, ISI histograms (bin size $=0.001 \mathrm{~s}$ ) were computed to confirm existence of harmonic modes in the beta/gamma range. $E$, Venn diagrams showing proportion of four different types of cells recorded in each structure. $F$, pie chart summarizing proportion of recording sites $(n=30)$ in which infra-slow, fast, co-expressed oscillations or none of them were observed. G, PSD for LFPs (left) and for an individual unit (right) recorded from the same recording channel. Note that while a narrowband oscillation is clearly detectable from the spiking activity of the unit no clear narrowband peak can be observed in LFPs. $H$, same as panel $G$ but for a different recording channel during the same experimental session. [Colour figure can be viewed at wileyonlinelibrary.com] 
Table 2. Cross-correlation between infra-slow oscillatory neurons, neurons co-expressing infra-slow and fast oscillations and between these two populations

\begin{tabular}{|c|c|c|c|c|c|c|c|c|c|}
\hline \multirow[t]{2}{*}{ Structure } & \multicolumn{3}{|c|}{ Infra-slow oscillations } & \multicolumn{3}{|c|}{$\begin{array}{c}\text { Co-expressed infra-slow and } \\
\text { fast oscillations }\end{array}$} & \multicolumn{3}{|c|}{ Between } \\
\hline & $n$ & $P$ value & $r$ & $N$ & $P$ value & $r$ & $n$ & $P$ value & $r$ \\
\hline \multicolumn{10}{|c|}{ Opn1mwR mice } \\
\hline $\mathrm{dLGN}$ & 420 & 0 & 0.238 & 27 & 0 & 0.370 & 82 & 0 & 0.187 \\
\hline vLGN & 40 & 0 & 0.159 & 11 & 0.065 & 0.106 & 7 & 0.5 & -0.162 \\
\hline OPN & 27 & 0.442 & 0.098 & 32 & 0 & 0.283 & 67 & 0.0002 & 0.118 \\
\hline Pretectum & 45 & 0.036 & 0.086 & 4 & 0.125 & 0.127 & 7 & 1 & -0.0005 \\
\hline \multicolumn{10}{|l|}{ Opn $4^{-1-}$ mice } \\
\hline $\mathrm{dLGN}$ & 89 & 0.0003 & 0.107 & 6 & 0.0312 & 0.310 & - & - & - \\
\hline vLGN & 37 & 0.099 & 0.096 & 5 & 0.375 & 0.213 & 21 & 1 & 0.079 \\
\hline OPN & 15 & 0.001 & 0.090 & 6 & 0.031 & 0.306 & - & - & - \\
\hline Pretectum & 33 & 1 & 0.042 & - & - & & - & - & - \\
\hline
\end{tabular}

Data were analysed by sign-test; $n$, number of correlated pairs of neurons; bin size $=1 \mathrm{~s}$. Abbreviations: OPN, olivary pretectal

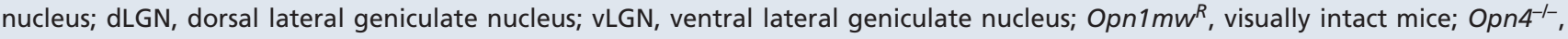
animals lacking melanopsin.

To address this question, we tested the possibility that co-expression of fast and infra-slow oscillations could simply occur by chance. The average firing rate of the four types of neurons were significantly different being the highest for units not expressing infra-slow but fast beta/gamma (Fig. 4G; Kruskal-Wallis test, $P=2.63 \mathrm{e}^{-35}$, $n=822$ ). However, the distributions of the four types as function of firing rate were largely overlapping (Fig. $4 H$ ) and this allowed us to perform a permutation test that preserved such distributions (see Permutation test in Methods and Table 1 for details). Therefore,we compared the observed number of units where the rhythms were co-expressed with the number we would expect under the null hypothesis that these two oscillations were independent (see Permutation test in Methods and Table 1 for details). We asked whether a statistical dependence between infra-slow and fast oscillations expressing neurons would emerge from the whole. To do so, we pooled together all neuronal groups recorded across structures. We found that the number of neurons co-expressing infra-slow and fast oscillations was larger than expected by chance, indicating that these rhythms tended to co-occur at the level of individual units (Fig. 4I).

\section{Outer and inner retina photoreception plays different roles in the control of infra-slow modulation of fast beta/gamma oscillations}

The data from visually intact mice thus suggest that infra-slow and fast oscillations are separable phenomena, which nonetheless co-occur more than expected by chance. Previous results indicate that expression of both infra-slow and fast beta/gamma oscillations depends both on outer and inner retina photoreception (respectively rods/cones and melanopsin) (Orlowska-Feuer et al. 2016a; Storchi et al. 2017; Chrobok et al. 2018). We now employed disruption of these two types of photoreceptors to further explore the inter-dependence of oscillations at high and infra-slow frequency. We repeated our initial experiments in a mouse model of aggressive retinal degeneration, lacking rod and cone photoreceptors at the time of recording $(r d / r d c l$; Lucas \& Foster, 1999; Lucas et al. 1999), and in mice with a more subtle deficit in retinal function caused by loss of the inner retinal photopigment melanopsin $\left(\mathrm{Opn} 4^{-/-}\right)$. The detailed analysis of all recorded and analysed cells in all genotypes is presented in Table 3. Importantly, there were no differences in the mean firing rates between genotypes across the structures investigated (dLGN: Kruskal-Wallis test, Kruskal-Wallis statistics $=5.901, P=0.0523$; vLGN: Kruskal-Wallis test, Kruskal-Wallis statistics $=2.011, P=0.3659$; OPN: Kruskal-Wallis test, Kruskal-Wallis statistics $=5.740$, $P=0.0567)$.

In both groups of mice we found significant differences in the prevalence and the features of both infra-slow and fast beta/gamma oscillations compared with visually intact animals. The highest percentage of units expressing infra-slow oscillations was observed in $r d / r d c l$ (41\%, 31\% and $56 \%$ for the dLGN, vLGN and OPN, respectively), followed by $O p n 1 m w^{R}(28 \%, 22 \%$ and $30 \%)$, and $O p n 4^{-/-}$ mice $(16 \%, 16 \%$ and $18 \%)$. Also, the frequency of infra-slow oscillations was significantly different among genotypes. Specifically both $r d / r d ~ c l$ and $O p n 4^{-/-}$mice exhibited faster infra-slow oscillations compared with visually intact animals; however, in the OPN that was only seen as a trend (dLGN: Kruskal-Wallis test, Kruskal-Wallis statistics $=37.53, P<0.0001$; vLGN: Kruskal-Wallis 
test, Kruskal-Wallis statistics $=11.05, P=0.0040$; OPN: Kruskal-Wallis test, Kruskal-Wallis statistics $=3.137$, $P=0.2084$; Fig. 5).

In $r d / r d c l$ mice, in which almost half of the recorded units exhibited infra-slow oscillatory activity, fast oscillations were largely abolished (dLGN: 5 out of 258; vLGN: 0 out of 100, OPN: 4 out of 89). On the other hand, in $O p n 4^{-/-}$mice the percentage of units expressing fast oscillations was comparable with visually intact animals
(7\%, $17 \%$ and $23 \%$ in the dLGN, vLGN and OPN, Fig. $5 I$ ). However, the frequency of fast oscillations was altered in $O p n 4^{-/-}$mice in the OPN (lower in the Opn $4^{-/-}$mice) and vLGN (higher in the Opn $4^{-/-}$mice), but not in dLGN (Fig. $5 H$ ).

Since both rhythms were retained following melanopsin loss, we next asked whether this manipulation had altered the interaction between infra-slow and fast beta/gamma oscillations. Similarly to what we found in
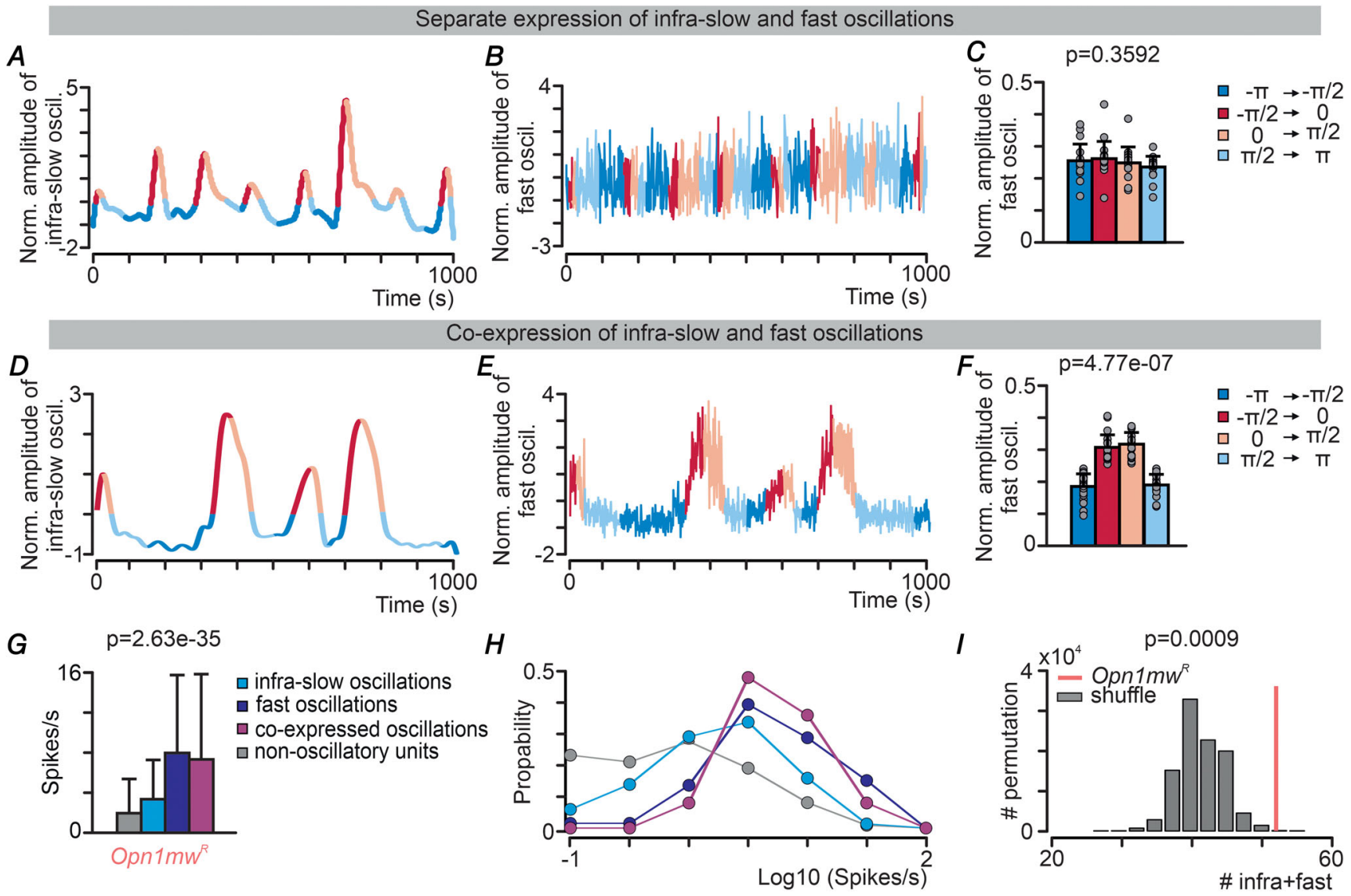

\begin{abstract}
Figure 4. Infra-slow modulation of fast beta/gamma oscillations in the subcortical visual system of Opn $1 m w^{R}$ mice

Representative example of simultaneously recorded activity from two separate pools of neurons. Firing rate activity has been filtered in the infra-slow $(A)$ and fast beta/gamma $(B)$ range and normalized as $z$-score. The colour of the lines denotes four different phases of the infra-slow rhythm according to the phase quadrants (dark blue: $-\pi$ to $-\pi / 2$ radians; dark red: $-\pi / 2$ to 0 radians; pink 0 to $\pi / 2$ radians; pale blue: $\pi / 2$ to $\pi$ radians). $C$, a non-parametric sign-test comparison between fast beta/gamma amplitude recorded during the 'red' vs the 'blue' phases shows no significant phase modulation. Here the data are grouped across all experiments in which distinct neuronal pools expressing either infra-slow or fast beta/gamma were simultaneously identified. $D$ and $E$ are the same as $A$ and $B$, respectively, but for a representative example in which a pool of simultaneously recorded neurons co-expressed both infra-slow and fast oscillations. $F$, same as panel $C$ but for all experiments in which a population of neurons co-expressing both rhythms was identified. Within these populations the amplitude of fast beta/gamma is significantly modulated by the phase of infra-slow oscillations. $G$, firing rates (mean \pm SD) for all the four unit types described in Fig. $2 A-E$ (Kruskal-Wallis test, $P=2.63 \mathrm{e}^{-35}, n=822$ ). $H$, distribution of firing rates across the four unit types. I, statistical comparison between the number of neurons co-expressing infra-slow and fast beta/gamma oscillations that we experimentally observed and the theoretical number predicted by the chance levels and based on the overall number of neurons expressing those two rhythms (either separately or jointly). The number of neurons in which infra-slow and fast beta/gamma are co-expressed (the red line) is larger than chance across our dataset. The null distribution (grey histogram) is obtained by using the permutation test as described in Methods section. [Colour figure can be viewed at wileyonlinelibrary.com]
\end{abstract}


Table 3. Number and proportion of all recorded neurons

\begin{tabular}{|c|c|c|c|c|c|c|c|c|c|}
\hline \multirow[b]{2}{*}{ Genotype } & \multicolumn{3}{|c|}{ No. of cells } & \multicolumn{3}{|c|}{ No. of infra-slow oscillatory cells } & \multicolumn{3}{|c|}{ No. of fast oscillatory cells } \\
\hline & Opn1mw $w^{R}$ & $\mathrm{rd} / \mathrm{rd} \mathrm{cl}$ & Opn $4^{-1-}$ & Opn1mw $w^{R}$ & $\mathrm{rd} / \mathrm{rd} \mathrm{cl}$ & Opn $4^{-1-}$ & Opn1mw $w^{R}$ & $\mathrm{rd} / \mathrm{rd} \mathrm{cl}$ & Opn $4^{-1-}$ \\
\hline \multirow[t]{2}{*}{$\mathrm{dLGN}$} & 400 & 258 & 340 & 111 & 107 & 53 & 44 & 5 & 23 \\
\hline & $(17 \pm 8)$ & $(23 \pm 10)$ & $(16 \pm 8)$ & $(5 \pm 6)$ & $(10 \pm 12)$ & $(3 \pm 2)$ & $(2 \pm 3)$ & $(0.5 \pm 0.8)$ & $(1 \pm 2)$ \\
\hline \multirow[t]{2}{*}{ vLGN } & 144 & 100 & 208 & 32 & 31 & 34 & 22 & 0 & 36 \\
\hline & $(8 \pm 7)$ & $(11 \pm 7)$ & $(10 \pm 8)$ & $(2 \pm 2)$ & $(3 \pm 5)$ & $(2 \pm 2)$ & $(2 \pm 3)$ & & $(2 \pm 3)$ \\
\hline \multirow[t]{2}{*}{ OPN } & 96 & 97 & 66 & 29 & 53 & 12 & 33 & 4 & 15 \\
\hline & $(14 \pm 5)$ & $(10 \pm 5)$ & $(9 \pm 6)$ & $(4 \pm 4)$ & $(5 \pm 5)$ & $(2 \pm 3)$ & $(5 \pm 2)$ & $(0.4 \pm 0.7)$ & $(2 \pm 3)$ \\
\hline \multirow{2}{*}{$\begin{array}{l}\text { Pretectum } \\
\text { (beyond } \\
\text { OPN) }\end{array}$} & 182 & 265 & 216 & 29 & 101 & 21 & 17 & 3 & 14 \\
\hline & $(23 \pm 17)$ & $(22 \pm 12)$ & $(27 \pm 8)$ & $(4 \pm 2)$ & $(8 \pm 7)$ & $(3 \pm 2)$ & $(2 \pm 2)$ & $(0.25 \pm 0.43)$ & $(2 \pm 1)$ \\
\hline \multicolumn{10}{|c|}{ Summary information } \\
\hline \multicolumn{2}{|c|}{ Genotype } & Opn1mw $w^{R}$ & $\mathrm{rd} / \mathrm{rd} \mathrm{cl}$ & Opn4 $4^{-1-}$ & \multicolumn{2}{|c|}{ Genotype } & Opn1mw $w^{R}$ & $\mathrm{rd} / \mathrm{rd} \mathrm{cl}$ & Opn4 $4^{-/-}$ \\
\hline \multirow[t]{3}{*}{$\begin{array}{l}\text { Number } \\
\text { of } \\
\text { animals }\end{array}$} & Total & 22 & 17 & 22 & $\begin{array}{l}\text { Number of } \\
\text { recorded/ } \\
\text { analysed } \\
\text { cells }\end{array}$ & Total & $936 / 822$ & $768 / 720$ & $902 / 830$ \\
\hline & OPN & 5 & 8 & 5 & & $\begin{array}{l}\text { OPN + } \\
\text { beyond }\end{array}$ & $300 / 278$ & $364 / 362$ & $312 / 282$ \\
\hline & LGN & 17 & 9 & 17 & & LGN & $636 / 544$ & $404 / 358$ & $590 / 548$ \\
\hline
\end{tabular}

Infra-slow and fast beta/gamma oscillatory activities presented in different structures of the mice subcortical visual system and in different genotypes. Mean number of cells \pm SD per recording site are presented in brackets. Abbreviations: OPN, olivary pretectal nucleus, LGN, lateral geniculate nucleus; dLGN, dorsal lateral geniculate nucleus; vLGN, ventral lateral geniculate nucleus; Opn1mw ${ }^{R}$, visually intact mice; $r d / r d ~ c l$, animals lacking rods and cones; $O p n 4^{-l}$, animals lacking melanopsin.

visually intact animals, neurons recorded from $O p n 4^{-/-}$ mice could be classified into four distinct types on the base of their expression of one or either oscillatory activity. All four types of neurons were found in $28 \%$ of recording sites while in $13 \%$ of recordings we did not find any oscillatory activity (in total 614 units from 32 recording sites and 22 animals were analysed). Consistent with the Opn1mw ${ }^{R}$ genotype, cross-correlation analysis revealed that in the dLGN firing rates were correlated within populations of neurons expressing only infra-slow or both rhythms (Table 2 for Opn $4^{-/-}$). However, different from visually intact animals, the firing rates were not correlated between these two populations (Table 2 for $O p n 4^{-/-}$). Infra-slow modulation of fast oscillations was observed, but as in visually intact mice, this only occurred in neurons expressing both rhythms (Fig. 6).

Finally we asked whether the rate of co-expression of both oscillations was also preserved. We found that this was not the case since co-expression of infra-slow and fast beta/gamma oscillations was not significantly different from chance level in $O p n 4^{-/-}$mice (Fig. 6G-I). This result indicates that inner retinal photoreception, while not required for co-existence of both rhythms, is necessary for tying these rhythms together and enabling a diffuse modulation of fast beta/gamma oscillations in the infra-slow range.

\section{Discussion}

Infra-slow oscillations have been ubiquitously observed in the subcortical visual system, but their function remains poorly understood. On the other hand, fast beta/gamma oscillations are involved in several types of visual processing that include inter-area communication (Fries, 2005) along the visual pathway and feature binding (Gray et al. 1989). It was previously shown that gamma rhythm couples with slower rhythms expressed in different brain areas (Vanhatalo et al. 2004; Monto et al. 2008), and thus we asked whether that is also the case with infra-slow oscillatory activity in the subcortical visual system.

We find that these two types of oscillation are distinct phenomena, but that they do co-occur more than expected by chance. Importantly, this co-occurrence allows the phase of infra-slow oscillations to modulate the amplitude of the fast. These findings reveal a 
potential mechanism by which infra-slow oscillations could regulate the visual flow - by modulating the amplitude of fast beta/gamma oscillations in the mouse visual system.

\section{Infra-slow oscillations are a common feature of subcortical visual systems}

Oscillations with the period of minutes were described before in the visual system of adult rats (SCN: Miller \& Fuller, 1992; dLGN: Albrecht \& Gabriel, 1994; IGL: Lewandowski et al. 2000; OPN: Szkudlarek et al. 2008), cats (optic chiasm: Cavaggioni, 1968; retina: Rodieck \& Smith, 1966; dLGN: Lorincz et al. 2009, Hughes et al. 2004), rabbits (visual cortex: Aladjalova, 1957), monkeys (visual cortex: Leopold et al. 2003; LGN: Cheong et al. 2011) and mice (prefrontal cortex, EEG recordings: Lecci et al. 2017; prefrontal cortex: Okun et al. 2019). Our study is the first to show the existence of infra-slow oscillatory rhythm in the mouse subcortical visual system, where we recorded them in the OPN and in both divisions of LGN. According to the present data, infra-slow oscillatory neurons constitute $\sim 30 \%$ of all recorded units in dark-adapted mice. The frequency of observed oscillations is slower $(\sim 0.005 \mathrm{~Hz})$ than those of rats $(\sim 0.01 \mathrm{~Hz})$; however, there is no doubt that the pattern of spike generation is the same (Fig. 2). Differences between these species in their frequency could have different potential origins. On one hand, it could be a
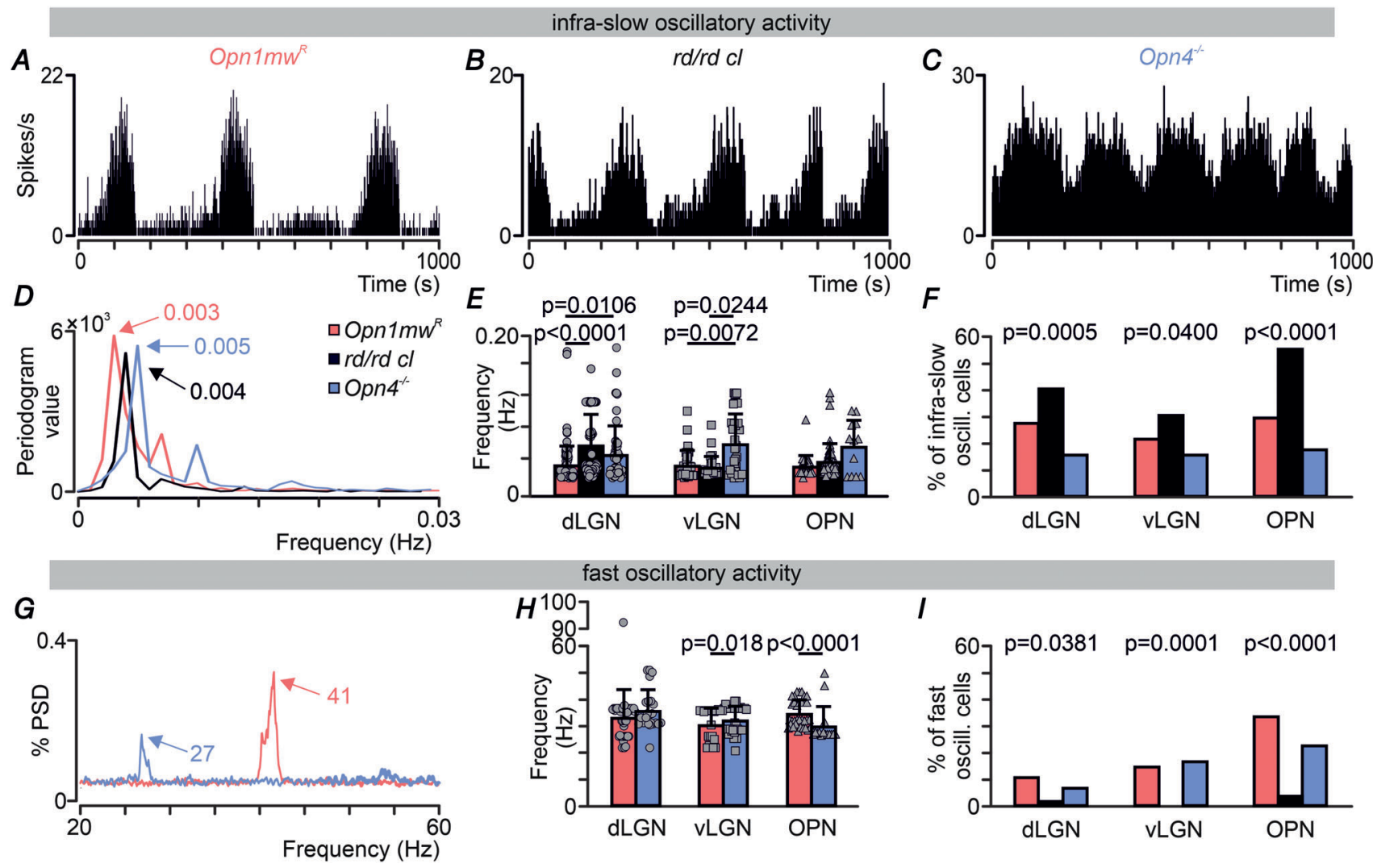

Figure 5. Inner and outer retina contribution to emergence of infra-slow and fast beta/gamma oscillatory rhythms

$A-C$, representative firing rate histograms (bin size $=1 \mathrm{~s}$ ) of infra-slow oscillatory activities recorded within the $\mathrm{dLGN}$

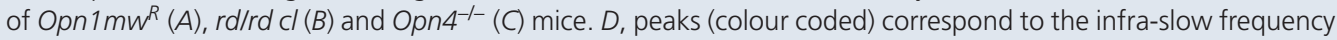
of oscillations presented on $A-C$, respectively. $E$, mean frequency of recorded infra-slow oscillations differs between genotypes in the dLGN and VLGN, but not in the OPN (however, the trend could be seen). $F$, the proportion of infra-slow oscillatory cells differs between the genotypes ( $\chi^{2}$ test; $\mathrm{dLGN}: P=0.0005, \chi^{2}$ statistic $=15.40, \mathrm{df}=2$; vLGN: $P=0.0400, \chi^{2}$ statistic $=6.437, \mathrm{df}=2 ; \mathrm{OPN}: P<0.0001, \chi^{2}$ statistic $\left.=33.32, \mathrm{df}=2\right) . \mathrm{G}$, normalized PSDs

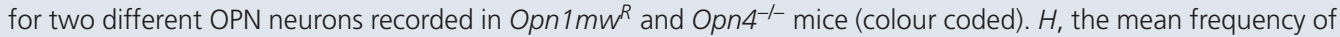
fast beta/gamma oscillating neurons differs between Opn $1 \mathrm{mw}^{R}$ and $O p n 4^{--}$in the OPN and vLGN, but not dLGN. I, the proportion of fast beta/gamma oscillatory cells differs between the genotypes $\left(\chi^{2}\right.$ test: dLGN: $P=0.0381, \chi^{2}$ statistic $=6.536, \mathrm{df}=2 ; \mathrm{vLGN}: P=0.0001, \chi^{2}$ statistic $=18.12, \mathrm{df}=2 ;$ OPN: $P<0.0001, \chi^{2}$ statistic $=28.44$, $\mathrm{df}=2$ ). Please note, that fast oscillatory cells were not recorded at all in $\mathrm{vLGN}$ in $r d / r d \mathrm{cl}$ animals. Data in $E$ analysed by Kruskal-Wallis test followed by Dunn's multiple comparison test; data in H analysed by Mann-Whitney test. [Colour figure can be viewed at wileyonlinelibrary.com] 
genuine species difference in visual physiology. On the other, it may be connected to the fact that albino Wistar rats carry dysfunctions in the visual system (Bolles \& Woods, 1964; Lund, 1964; Heiduschka \& Schraermeyer, 2008; Nadal-Nicolás et al. 2012) while this study was performed in fully pigmented mice. Finally, it is possible that part of the variability could be simply explained by the small sample sizes used in the previous studies, where single channel recordings were employed as opposed to the higher-yield multichannel electrodes used in this study.

Infra-slow and fast beta-gamma oscillations interact through co-expression of both rhythms at the level of single units

While co-existence of infra-slow and fast beta/gamma has been reported in the subcortical visual system (Tsuji et al. 2016; Chrobok et al. 2018), until now the extent to which they are independent events and whether they interact was unknown. Our data, obtained by unbiased recordings of all active neurons in the dark, reveal that while a subset of cells express only one type of oscillation, others express either both or none (Fig. 3).

In principle, in such population in which not all neurons expressing one type of oscillation also have the other, infra-slow oscillations could still modulate fast beta/gamma oscillations by two types of circuitries. On the one hand, the population of infra-slow neurons could provide a modulation of beta/gamma oscillations, including neurons that did not themselves have infra-slow oscillations (Fig. 7A-F). Alternatively, such modulation could be restricted to neurons in which the two types of oscillations could interact within single neurons (Fig. $7 \mathrm{H}-J$ ).

Our analyses revealed that only neurons co-expressing both oscillations are responsible for mediating infra-slow modulation of fast beta/gamma oscillations (Fig. $4 D-F$ ). That is, when they co-occur the amplitude of fast modulations is modified according to the infra-slow rhythm. Importantly, the level of co-expression of these rhythms was higher than expected by two independent events, suggesting that the interaction between infra-slow and fast beta/gamma occurs 'by design' rather than simply by chance (Fig. $4 G$ ).
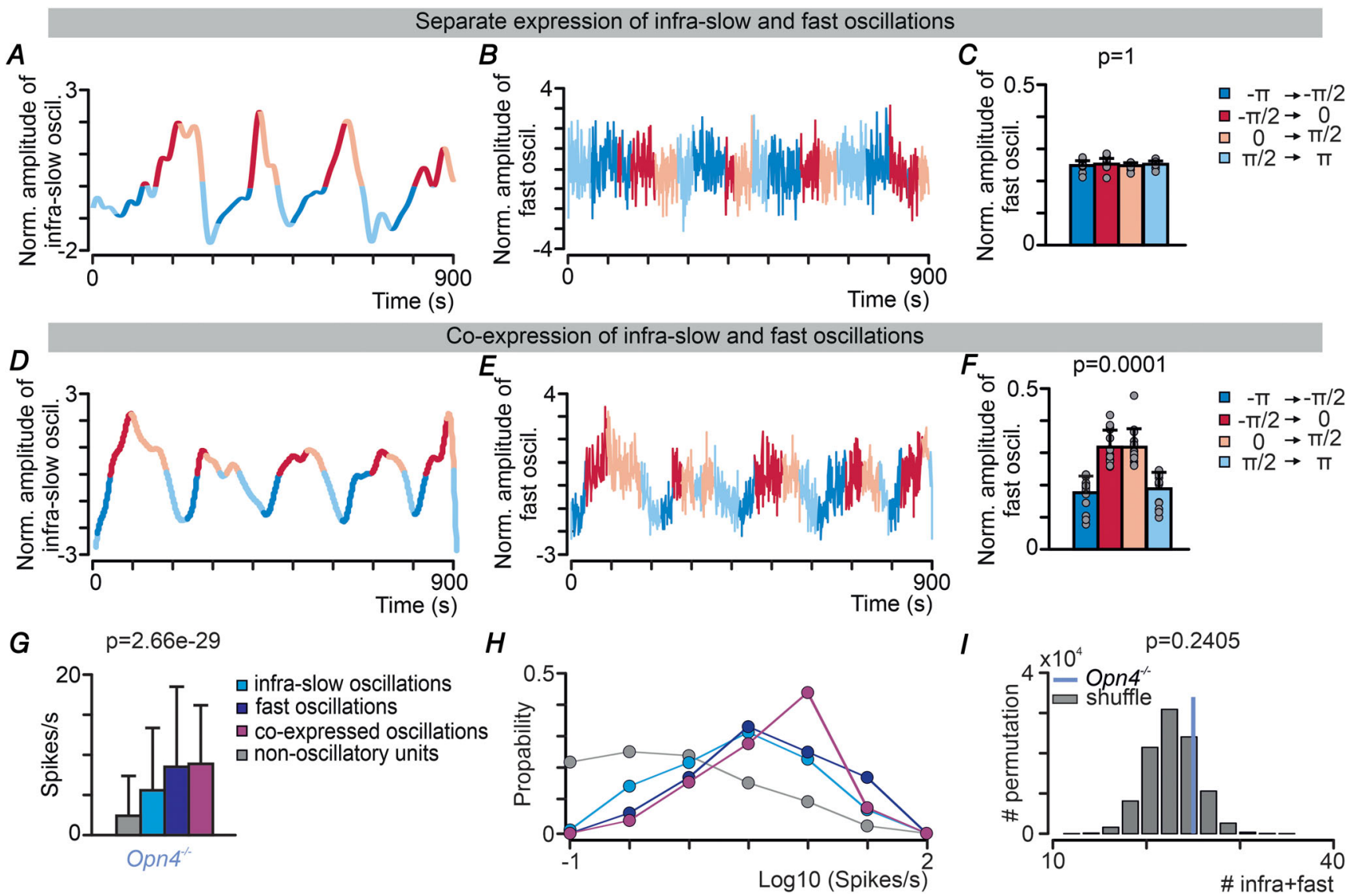

Figure 6. Infra-slow modulation of fast beta/gamma oscillations in the subcortical visual system of Opn4 ${ }^{-1-}$ mice Same as Fig. 4 but for the Opn $4^{-/-}$dataset. [Colour figure can be viewed at wileyonlinelibrary.com] 
It is currently not possible to map the circuits displayed in Fig. 7 onto specific anatomical connections since the infra-slow modulation could be inherited by the retina or generated at the level of the subcortical visual system. Further in vivo studies performing simultaneous recordings from optic nerve and from neurons in the subcortical visual system would be helpful to clarify the specific circuits involved.
Changes to the retinal network alter appearance and interaction of infra-slow and fast oscillations

The retina has been previously implicated in the emergence of both infra-slow and fast oscillations in the subcortical brain, with intraocular injection of tetradotoxin abolishing both (Lewandowski \& Błasiak, 2004; Szkudlarek et al. 2012; Chrobok et al. 2018). Moreover, selective blockade of phototransduction
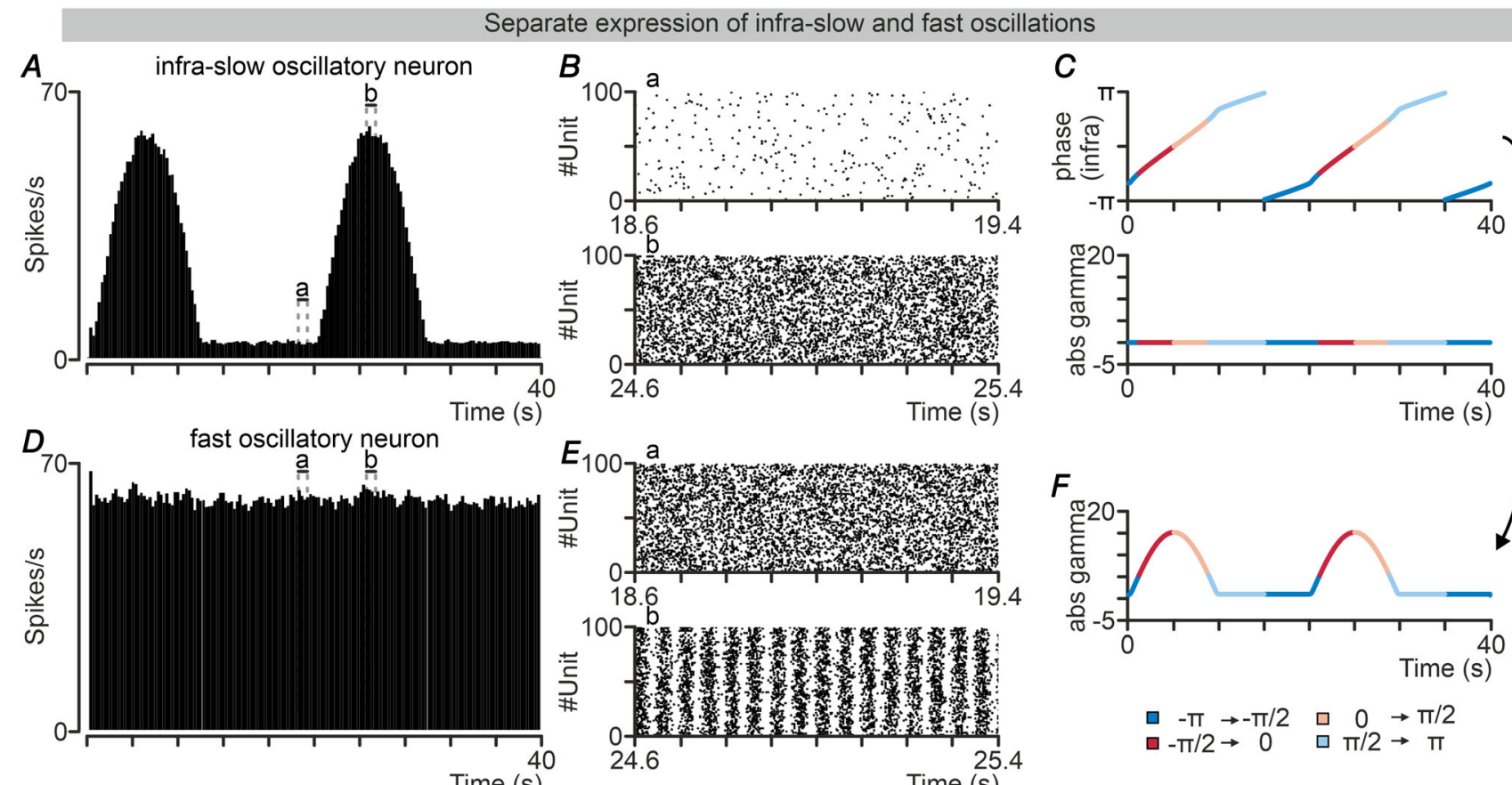

Time (s)

Time (s)

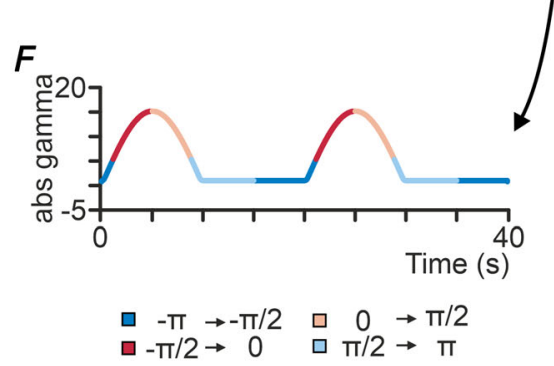

Co-expression of infra-slow and fast oscillations
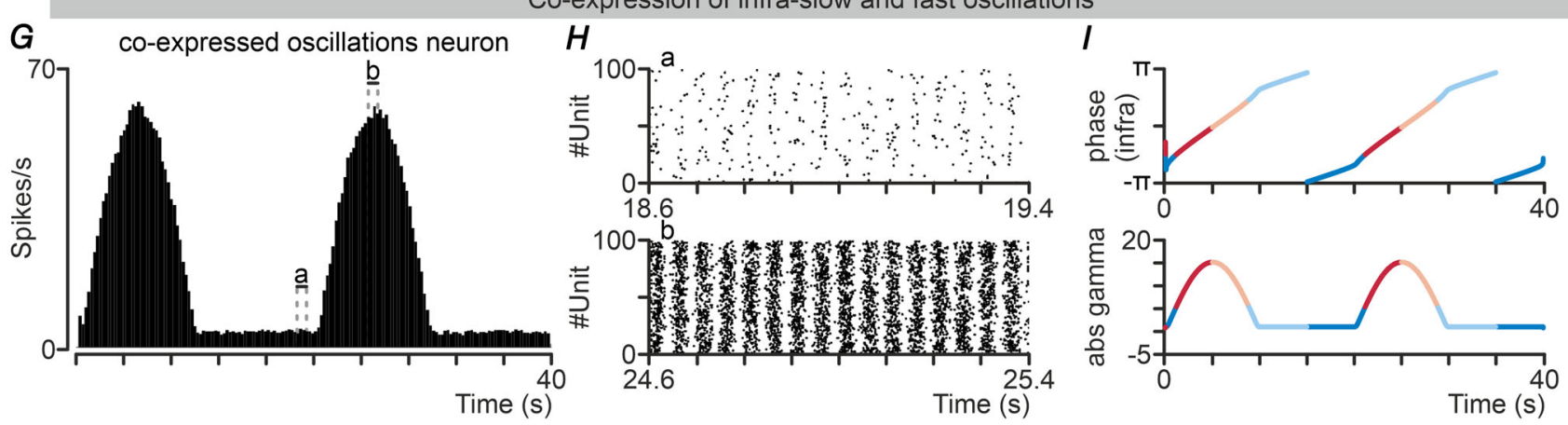

Figure 7. Potential circuits mediating infra-slow modulation of fast beta/gamma oscillations

$A-F$, the amplitude of fast/beta gamma oscillations is modulated according to a distinct but functionally connected population expressing infra-slow oscillations. In this way coherence of oscillations in the beta/gamma range is modulated by the phase of infra-slow oscillations. $A-C$, simulated firing pattern of a neuronal population expressing infra-slow oscillations $(A)$ and magnified single units activity at different phase of the infra-slow oscillations $(B)$. The population expresses changes in phase in the infra-slow range $(C$, top panel) but no fast beta/gamma oscillations ( $C$, bottom panel). $D-F$, a second neuronal population lacks infra-slow modulation of firing rate $(D)$ but exhibits epochs of regular fast beta/gamma oscillations ( $E$, bottom) interleaved with epochs lacking such fast rhythms $(E$, top). The amplitude of fast-beta gamma oscillations is coupled to the phase of infra-slow activity $(F)$. $G$, simulated firing of a neuronal population expressing both infra-slow and fast beta/gamma oscillations. $H$, magnified single units activity at different phase of the infra-slow oscillations. Clear beta/gamma oscillations can be observed during epochs of high firing rates. I, within the same population the amplitude of fast-beta gamma oscillations is coupled to the phase of infra-slow activity. [Colour figure can be viewed at wileyonlinelibrary.com] 
pathways (classic photoreceptors: rods and cones and melanopsin), as well as retinal gap junction blockade, were shown to disrupt infra-slow oscillatory rhythm (Orlowska-Feuer et al. 2016a,b). In all of the data cited above, intraocular injections were performed to assess retina contribution to the generation of infra-slow oscillatory activity in the subcortical visual system. However, such an approach cannot distinguish between a driving effect, in which infra-slow oscillations in the retina are directly inherited by sub-cortical nuclei, and a gating effect, in which excitation by the retina enables subcortical oscillations to emerge. Here, we studied rhythmicity in two mice strains in which the optic nerve was intact and active but in which retinal function was genetically disrupted. These were a model of aggressive photoreceptor degeneration, lacking rod+cone cells $(r d / r d c l$ mice), and animals in which inner retina photoreception is ablated (Opn $4^{-/-}$mice). As we were recording activity in the dark, the loss of photoreception in these manipulations is not itself expected to have an impact on our findings, but secondary effects on the retinal circuitry could. Photoreceptors degeneration has knock-on effects on inner-retinal neurons and induces aberrant spontaneous ganglion cell activity in the form of $\sim 10 \mathrm{~Hz}$ oscillations (Jones et al. 2003; Ryu et al. 2010; Menzler \& Zeck, 2011; Trenholm \& Awatramani, 2015), while melanopsin knockout has been reported to affect aspects of retinal development (Renna et al. 2011; Rao et al. 2013; Kirkby \& Feller, 2013).

Infra-slow oscillations were retained in all genotypes. In fact, they were most prevalent in the $r d / r d c l$ (see Table 3), consistent with the simulated effect of reduced retinal input (Zierenberg et al. 2018). Both genetic modifications altered their frequency, and this effect was replicated across all visual centres, consistent with the view that these infra-slow oscillations share a common mechanistic origin.

The situation with respect to fast beta/gamma oscillations was quite different, with this behaviour essentially absent in $r d / r d c l$ mice. This dramatic effect highlights the importance of the retinal activity for this rhythm. Since fast beta/gamma oscillations are typically network phenomena (Buzsáki \& Wang, 2012), that could be because the retinal circuits generate these oscillations (Kenyon et al. 2003), the interplay between central and retinal circuits is required to produce this rhythmicity; or because the change in retinal input to the brain alters the activity of central circuits generating this rhythmicity.

Perhaps the most interesting alteration in oscillatory activity in transgenic mice was the effective decoupling of infra-slow and fast oscillations in melanopsin knockout animals. While in intact animals these two rhythms co-occurred in individual neurons more often than by chance, this property was lost in the $\mathrm{Opn4^{-/- }}$ genotype. Although, we can only speculate as to the change in retinal circuitry responsible for this disruption, it does reinforce the conclusion that the co-occurrence of these oscillations in visually intact animals is an active process. It has been shown that vascular patterning of the retina is dependent upon intact melanopsin photoreception, since this process is disrupted in both dark-reared visually intact animals and in animals lacking melanopsin photoreception (Rao et al. 2013). Since vascular patterning affects development of neuronal networks in the retina, this could also affect the coupling between infra-slow and fast narrowband rhythms. This result also provides a new potential mechanism via which this inner retinal photoreceptor typically associated with subconscious reflex responses to light could contribute to conventional visual function. While it has been previously shown that the psychophysical threshold of visual sensitivity oscillates in the infra-slow range (Thoss et al. 1998) the neuronal mechanism underlying this phenomenon is currently unknown. The reported coupling between infra-slow fluctuations and fast beta/gamma oscillations, the latter being involved in visual processing, points towards such a potential mechanism.

\section{Limitations of the study}

Since the presented results were obtained from urethane-anesthetized mice an open question is to what extent those results generalize to awake behaving animals. While a number of studies have reported infra-slow and beta/gamma oscillations in various species under different experimental conditions e.g. anaesthetics (such as barbiturates, isoflurane) and recording procedures (LFPs, EEG, in vitro and in vivo neuronal recordings) (Albrecht et al. 1998; Filippov \& Frolov, 2005; Hughes et al. 2011; Storchi et al. 2017) some studies have also reported such rhythms in the dLGN and primary visual cortex in behaving animals (Albrecht et al. 1998; Filippov \& Frolov, 2005; Saleem et al. 2017; Storchi et al. 2017). The frequency of both types of oscillations was higher in freely moving compared to anaesthetized animals (Albrecht et al. 1998; Storchi et al. 2017) and such difference could be due to the specific action of the anaesthetics on neurotransmission systems (Hara \& Harris, 2002; Wu et al. 2004; Hemmings et al. 2005). Coupling between infra-slow and fast beta/gamma oscillations has also been recorded in awake cortical recordings in humans (Monto et al. 2008) but, to the best of our knowledge, never in the subcortical visual structures where our recordings were performed.

All recordings in the present study were performed under dark-adapted condition. It has been previously shown that both, infra-slow and beta/gamma oscillatory neurons are modulated by light and visual stimuli. Photopic backgrounds increase mean firing rate but not 
oscillatory frequency in neurons expressing infra-slow oscillations (Szkudlarek et al. 2012; Chrobok et al. 2018, however, see also Albrecht et al. 1998). In neurons expressing narrowband gamma oscillations both amplitude and frequency of such oscillations are enhanced by steady light (Saleem et al. 2017; Storchi et al. 2017). Those previous results indicate that bright light strongly increases the expression of fast narrowband oscillations and, consistent with this notion, expression of fast beta/gamma oscillations under dark-adapted conditions is limited to a minority of neurons. While individual units can still express narrowband oscillations such oscillations were not detectable in LFP recordings. This result is in accordance with previous studies indicating that the coupling between LFPs and spikes only becomes apparent with high synchrony at population level (Denker et al. 2011; Storchi et al. 2012). During spontaneous activity, synchrony is strongly reduced in comparison with evoked sensory responses and, as a result, coupling between and between LFP and spikes and between LFPs from different subcortical areas is also reduced (Storchi et al. 2012; Zippo et al. 2013).

\section{A potential role for the interaction between infra-slow and fast beta/gamma oscillations}

In considering the potential function of these two types of oscillation it is important to remember that they have very different spatiotemporal topology. Infra-slow fluctuations are sustained events that can last minutes/hours and are correlated between different brain areas (Szkudlarek et al. 2008; Chrobok et al. 2018). Infra-slow oscillations have also been closely associated with resting state networks (RSNs) observed in human fMRI recordings (Damoiseaux et al. 2006; De Luca et al. 2006; He et al. 2008), and most likely reflect the underlying structural connectivity (Fox \& Raichle, 2007; Honey et al. 2007). Fast beta/gamma oscillations typically are short-lasting events that can arise locally and transiently synchronize distant brain areas (Buzsáki, 2006). Those oscillations can be evoked by sensory stimulation (Gray et al. 1989; Cardin et al. 2009; Storchi et al. 2017) or by cognitive tasks (Fries, 2005) but can also emerge spontaneously (Tsuji et al. 2016; Chrobok et al. 2018; Foik et al. 2018). Thus, their spatiotemporal topology is more flexible and need not necessarily reflect the underlying structural connectivity. Accordingly, the interaction between infra-slow and fast beta/gamma could represent a 'balancing act' between the opposing needs for transient 'on-demand' flexibility served by fast beta/gamma oscillations - and long-term structural and dynamical stability - served by infra-slow oscillations.

Viewed from this perspective, the strong modulation of fast beta/gamma amplitude by infra-slow oscillations observed here could represent a mechanism to oppose runaway plasticity (Abbott \& Nelson, 2000) and promote the stability of visual signalling. This control would then be significantly reduced in $O p n 4^{-/-}$animals since these two rhythms are independently expressed within the subcortical visual networks. Consistent with this possibility a previous work showed that light modulation of fast beta/gamma oscillations is substantially reduced in $\mathrm{Opn}^{-/-}$animals (Storchi et al. 2017). Thus, although fast beta/gamma could be observed in the dLGN of Opn $4^{-/-}$ animals, these oscillations were less controllable by the visual stimulus. This lack in controllability could be due to the lack of the stabilizing action of infra-slow oscillations.

\section{References}

Abbott LF \& Nelson SB (2000). Synaptic plasticity: taming the beast. Nat Neurosci 3, 1178-1183.

Aggelopoulos NC \& Meissl H (2000). Responses of neurones of the rat suprachiasmatic nucleus to retinal illumination under photopic and scotopic conditions. J Physiol 523, 211-222.

Aladjalova NA (1957). Infra-slow rhythmic oscillations of the steady potential of the cerebral cortex. Nature 179, 957-959.

Albrecht D \& Gabriel S (1994). Very slow oscillations of activity in geniculate neurons of urethane anaesthetized rats. Neuroreport 5, 1909-1912.

Albrecht D, Royl G \& Kaneoke Y (1998). Very slow oscillatory activities in lateral geniculate neurons of freely moving and anesthetized rats. Neurosci Res 32, 209-220.

Allen AE, Brown TM \& Lucas RJ (2011). A distinct contribution of short-wavelength-sensitive cones to light-evoked activity in the mouse pretectal olivary nucleus. J Neurosci 31, 16833-16843.

Bolles RC \& Woods PJ (1964). The ontogeny of behaviour in the albino rat. Anim Behav 12, 427-441.

Brown TM, Gias C, Hatori M, Keding SR, Semo M, Coffey PJ, Gigg J, Piggins HD, Panda S \& Lucas RJ (2010). Melanopsin contributions to irradiance coding in the thalamo-cortical visual system. PLoS Biol 8, e1000558. https://doi.org/10. 1371/journal.pbio.1000558.

Buzsáki G (2006). Rhythms of the Brain.: Oxford University Press, USA.

Buzsáki G \& Wang X-J (2012). Mechanisms of gamma oscillations. Annu Rev Neurosci 35, 203-225.

Cardin JA, Carlén M, Meletis K, Knoblich U, Zhang F, Deisseroth K, Tsai LH \& Moore CI (2009). Driving fast-spiking cells induces gamma rhythm and controls sensory responses. Nature 459, 663-667.

Cavaggioni A (1968). The dark-discharge of the eye in the unrestrained cat. Pflügers Arch Eur J Physiol 304, 75-80.

Cheong SK, Tailby C, Martin PR, Levitte JB \& Solomona SG (2011). Slow intrinsic rhythm in the koniocellular visual pathway. Proc Natl Acad Sci U S A 108, 14659-14663.

Chrobok L, Palus-Chramiec K, Jeczmien-Lazur JS, Blasiak T \& Lewandowski MH (2018). Gamma and infra-slow oscillations shape neuronal firing in the rat subcortical visual system. J Physiol 596, 2229-2250. 
Damoiseaux JS, Rombouts SARB, Barkhof F, Scheltens P, Stam CJ, Smith SM \& Beckmann CF (2006). Consistent resting-state networks across healthy subjects. Proc Natl Acad Sci U S A 103, 13848-13853.

De Luca M, Beckmann CF, De Stefano N, Matthews PM \& Smith SM (2006). fMRI resting state networks define distinct modes of long-distance interactions in the human brain. Neuroimage 29, 1359-1367.

Denker M, Linde H, Diesmann M, Riehle A \& Gru S (2011). The local field potential reflects surplus spike synchrony. Cereb Cortex 21, 2681-2695.

Filippov IV \& Frolov VA (2005). Very slow potentials in the lateral geniculate complex and primary visual cortex during different illumination changes in freely moving rats. Neurosci Lett 373, 51-56.

Filippov IV, Williams WC \& Frolov VA (2004). Very slow potential oscillations in locus coeruleus and dorsal raphe nucleus under different illumination in freely moving rats. Neurosci Lett 363, 89-93.

Foik AT, Ghazaryan A \& Waleszczyk WJ (2018). Oscillations in spontaneous and visually evoked neuronal activity in the superficial layers of the cat's superior colliculus. Front Syst Neurosci 12, 1-19.

Fox MD \& Raichle ME (2007). Spontaneous fluctuations in brain activity observed with functional magnetic resonance imaging. Nat Rev Neurosci 8, 700-711.

Fries P (2005). A mechanism for cognitive dynamics: Neuronal communication through neuronal coherence. Trends Cogn Sci 9, 474-480.

Gray CM, Konig P, Engel AK \& Singer W (1989). Oscillatory responses in cat visual cortex exhibit inter-columnar global stimulus properties. Nature 338, 334-337.

Grundy D (2015). Principles and standards for reporting animal experiments in The Journal of Physiology and Experimental Physiology. J Physiol 593, 2547-2549.

Gupta A, van der Meer M, Touretzky D \& Redish D (2016). Segmentation of spatial experience by hippocampal theta sequences. Nat Neurosci 15, 1032-1039.

Hara K \& Harris A (2002). The anesthetic mechanism of urethane: the effects on neurotransmitter-gated ion channels. Anesth Pharmacol 313-318.

He BJ, Snyder AZ, Zempel JM, Smyth MD \& Raichle ME (2008). Electrophysiological correlates of the brain's intrinsic large-scale functional architecture. Proc Natl Acad Sci U S A 105, 16039-16044.

Heiduschka P \& Schraermeyer U (2008). Comparison of visual function in pigmented and albino rats by electroretinography and visual evoked potentials. Graefes Arch Clin Exp Ophthalmol 246, 1559-1573.

Hemmings HCJ, Akabas MH, Goldstein PA, Trudell JR, Orser BA \& Harrison NL (2005). Emerging molecular mechanisms of general anesthetic action. Trends Pharmacol Sci 26, 503-510.

Honey CJ, Kötter R, Breakspear M \& Sporns O (2007). Network structure of cerebral cortex shapes functional connectivity on multiple time scales. Proc Natl Acad Sci U S A 104, 10240-10245.
Hughes SW, Lörincz M, Cope DW, Blethyn KL, Kékesi KA, Parri HR, Juhász G \& Crunelli V (2004). Synchronized oscillations at $\alpha$ and $\theta$ frequencies in the lateral geniculate nucleus. Neuron 42, 253-268.

Hughes SW, Lőrincz ML, Parri HR \& Crunelli V (2011). Infraslow $(<0.1 \mathrm{~Hz})$ oscillations in thalamic relay nuclei mechanisms and significance to health and disease states. Prog Brain Res 193, 145-162.

Jacobs GH \& Williams GA (2007). Contributions of the mouse UV photopigment to the ERG and to vision. Doc Ophthalmol 115, 137-144.

Jones BW, Watt CB, Frederick JM, Baehr W, Chen CK, Levine EM, Milam AH, Lavail MM \& Marc RE (2003). Retinal remodeling triggered by photoreceptor degenerations. $J$ Comp Neurol 464, 1-16.

Kenyon GT, Moore B, Jeffs J, Denning KS, Stephens GJ, Travis BJ, George JS, Theiler J \& Marshak DW (2003). A model of high-frequency oscillatory potentials in retinal ganglion cells. Vis Neurosci 20, 465-480.

Kirkby LA \& Feller MB (2013). Intrinsically photosensitive ganglion cells contribute to plasticity in retinal wave circuits. Proc Natl Acad Sci U S A 110, 12090-12095.

Lecci S, Fernandez LMJ, Weber FD, Cardis R, Chatton JY, Born J \& Lüthi A (2017). Coordinated infraslow neural and cardiac oscillations mark fragility and offline periods in mammalian sleep. Sci Adv 3, e1602026; https://doi.org/10. 1126/sciadv.1602026.

Leopold DA, Murayama Y \& Logothetis NK (2003). Very slow activity fluctuations in monkey visual cortex: implications for functional brain imaging. Cereb Cortex 13, 422-433.

Lewandowski MH \& Błasiak T (2004). Slow oscillation circuit of the intergeniculate leaflet. Acta Neurobiol Exp 64, 277-288.

Lewandowski MH, Błasiak T \& Błasiak A (2002). Are ultra-slow isoperiodic oscillations in rat intergeniculate leaflet neurons dependent on reciprocal connection with its contralaterally located counterpart? Neurosci Lett 330, 243-246.

Lewandowski MH, Błasiak T, Domosławski J \& Wołkowska A (2000). Ultradian rhythmic neuronal oscillation in the intergeniculate leaflet. Neuroreport 11, 317-321.

Lisman JE \& Jensen O (2013). The theta-gamma neural code. Neuron 77, 1002-1016.

Lorincz ML, Geall F, Bao Y, Crunelli V \& Hughes SW (2009). ATP-dependent infra-slow $(<0.1 \mathrm{~Hz})$ oscillations in thalamic networks. PLoS One 4, e4447; https://doi.org/10. 1371/journal.pone.0004447.

Lucas RJ \& Foster RG (1999). Neither functional rod photoreceptors nor rod or cone outer segments are required for the photic inhibition of pineal melatonin. Endocrinology 140, 1520-1524.

Lucas RJ, Freedman MS, Muñoz M, Garcia-Fernández JM \& Foster RG (1999). Regulation of the mammalian pineal by non-rod, non-cone, ocular photoreceptors. Science 284, 505-507.

Lund RD (1964). Uncrossed visual pathways of hooded and albino rats. Science 149, 1964-1965. 
Menzler J \& Zeck G (2011). Network oscillations in rod-degenerated mouse retinas. J Neurosci 31, 2280-2291.

Miller JD \& Fuller CA (1992). Isoperiodic neuronal activity in suprachiasmatic nucleus of the rat. Am J Physiol Regul Integr Comp Physiol 263, R51-R58.

Monto S, Palva S, Voipio J \& Palva JM (2008). Very slow EEG fluctuations predict the dynamics of stimulus detection and oscillation amplitudes in humans. J Neurosci 28, 82688272.

Nadal-Nicolás FM, Jiménez-López M, Salinas-Navarro M, Sobrado-Calvo P, Alburquerque-Béjar JJ, Vidal-Sanz M \& Agudo-Barriuso M (2012). Whole number, distribution and co-expression of brn 3 transcription factors in retinal ganglion cells of adult albino and pigmented rats. PLoS One 7, e49830; https://doi.org/10.1371/journal.pone.0049830.

Okun M, Steinmetz NA, Lak A, Dervinis M \& Harris KD (2019). Distinct structure of cortical population activity on fast and infraslow timescales. Cereb Cortex 29, 2196-2210.

Orlowska-Feuer P, Allen AE, Storchi R, Szkudlarek HJ \& Lewandowski MH (2016a). The contribution of inner and outer retinal photoreceptors to infra-slow oscillations in the rat olivary pretectal nucleus. Eur J Neurosci 43, 823833.

Orlowska-Feuer P, Jeczmien-Lazur JS, Szkudlarek HJ \& Lewandowski MH (2016b). Retinal gap junctions are involved in rhythmogenesis of neuronal activity at remote locations - Study on infra-slow oscillations in the rat olivary pretectal nucleus. Neuroscience 339, 150-161.

Paxinos G \& Franklin K (2004). The Mouse Brain in Stereotaxic Coordinates, 2nd edn. Gulf Professional, San Diego, CA, USA.

Rao S, Chun C, Fan J, Kofron JM, Yang MB, Hegde RS, Ferrara N, Copenhagen DR \& Lang RA (2013). A direct and melanopsin-dependent fetal light response regulates mouse eye development. Nature 494, 243-246.

Renna J, Weng S \& Berson D (2011). Light acts through melanopsin to alter retinal waves and segregation of retinogeniculate afferents. Nat Neurosci 14, 827-829.

Rodieck R \& Smith P (1966). Slow dark discharge rhythms of cat retinal ganglion cells. J Neurophysiol 29, 942-953.

Ryu SB, Ye JH, Goo YS, Kim CH \& Kim KH (2010). Temporal response properties of retinal ganglion cells in rd1 mice evoked by amplitude-modulated electrical pulse trains. Investig Ophthalmol Vis Sci 51, 6762-6769.

Saleem AB, Lien AD, Krumin M, Haider B, Rosón MR, Ayaz A, Reinhold K, Busse L, Carandini M, Harris KD \& Carandini M (2017). Subcortical source and modulation of the narrowband gamma oscillation in mouse visual cortex. Neuron 93, 315-322.

Smallwood PM, Olveczky BP, Williams GL, Jacobs GH, Reese BE, Meister M \& Nathans J (2003). Genetically engineered mice with an additional class of cone photoreceptors: implications for the evolution of color vision. Proc Natl Acad Sci U S A 100, 11706-11711.

Storchi R, Bedford RA, Martial FP, Allen AE, Wynne J, Montemurro MA, Petersen RS \& Lucas RJ (2017). Modulation of fast narrowband oscillations in the mouse retina and dLGN according to background light intensity. Neuron 93, 299-307.
Storchi R, Zippo AG, Caramenti GC, Valente M \& Biella GEM (2012). Predicting spike occurrence and neuronal responsiveness from LFPs in primary somatosensory cortex. PloS One 7, e35850. https://doi.org/10.1371/journal.pone. 0035850.

Szkudlarek HJ, Herdzina O \& Lewandowski MH (2008). Ultra-slow oscillatory neuronal activity in the rat olivary pretectal nucleus: comparison with oscillations within the intergeniculate leaflet. Eur J Neurosci 27, 2657-2664.

Szkudlarek HJ, Orlowska P \& Lewandowski MH (2012). Light-induced responses of slow oscillatory neurons of the rat olivary pretectal nucleus. PLoS One 7, e33083.

Teich MC, Heneghan C, Lowen SB, Ozaki T \& Kaplan E (1997). Fractal character of the neural spike train in the visual system of the cat. J Opt Soc Am A 14, 529-545.

Thoss F, Bartsch B \& Stebel J (1998). Analysis of oscillations of the visual sensitivity. Vision Res 38, 139-142.

Trenholm S \& Awatramani GB (2015). Origins of spontaneous activity in the degenerating retina. Front Cell Neurosci $\mathbf{9}$, $1-7$.

Tsuji T, Tsuji C, Ludwig M \& Leng G (2016). The rat suprachiasmatic nucleus: the master clock ticks at $30 \mathrm{~Hz}$. J Physiol 594, 3629-3650.

Vanhatalo S, Palva JM, Holmes MD, Miller JW, Voipio J \& Kaila K (2004). Infraslow oscillations modulate excitability and interictal epileptic activity in the human cortex during sleep. Proc Natl Acad Sci U S A 101, 5053-5057.

Wu X, Ph D, Sun J, Ph D, Evers AS, Crowder M \& Ph D (2004). Isoflurane inhibits transmitter release and the presynaptic action potential. Anesthesiology 100, 663-670.

Zierenberg J, Wilting J \& Priesemann V (2018). Homeostatic plasticity and external input shape neural network dynamics. Phys Rev X 8, 31018.

Zippo AG, Storchi R, Nencini S, Caramenti GC, Valente M \& Biella EM (2013). Neuronal functional connection graphs among multiple areas of the rat somatosensory system during spontaneous and evoked activities. PLoS Comput Biol 9, e1003104; https://doi.org/10.1371/journal.pcbi. 1003104.

\section{Additional information}

\section{Data availability statement}

The data that support the findings of this study are openly available in github at https://github.com/RStorchi/InfraSlow.

\section{Competing interests}

The authors declare no competing interests.

\section{Author contributions}

P.O.-F., H.J.S., R.J.L., R.S. designed research; P.O.-F., A.E.A., T.M.B. performed research; R.S. contributed analytic tools; P.O.-F., H.J.S., R.S. analysed data; P.O.-F., R.J.L., R.S. interpreted the results; P.O.-F., R.J.L., R.S. wrote the paper. All authors have approved the final version of the manuscript and agreed to be 
accountable for all aspects of the work in ensuring that questions related to the accuracy or integrity of any part of the work are appropriately investigated and resolved. All persons designated as authors qualify for authorship and all those who qualify for authorship are listed.

\section{Funding}

The work of POF was supported by the Bekker Programme implemented by the Polish National Agency for Academic Exchange (NAWA). R.S. was funded by National Centre for Replacement Refinement and Reduction of Animals in Research (NC3Rs) via a David Sainsbury Fellowship (NC/P001505/1). R.J.L. was funded by The Welcome Trust (Investigator
Award 210684/Z/18/Z) and The European Research Council (268970).

\section{Keywords}

beta/gamma oscillations, infra-slow oscillations, in vivo electrophysiology, photoreceptors, subcortical visual system

\section{Supporting information}

Additional supporting information may be found online in the Supporting Information section at the end of the article.

\section{Statistical Summary Document}

